\title{
Thirty Years of Cometary Spectroscopy from McDonald Observatory
}

\author{
A. L. Cochran ${ }^{\mathrm{a}, *}$, E. S. Barker ${ }^{\mathrm{b}}$, C. L. Gray ${ }^{\mathrm{c}}$ \\ ${ }^{a}$ McDonald Observatory, University of Texas, 1 University Station C1402, Austin, TX 78712 \\ ${ }^{b}$ LZ Technology, Inc., 1110 NASA Parkway Suite 650, Houston, TX 77058 \\ ${ }^{c}$ New Mexico State University, 1320 Frenger Street, Las Cruces, NM 88003
}

\begin{abstract}
We report on the results of a spectroscopic survey of 130 comets that was conducted at McDonald observatory from 1980 through 2008. Some of the comets were observed on only one night, while others were observed repeatedly. For 20 of these comets, no molecules were detected. For the remaining 110 comets, some emission from $\mathrm{CN}, \mathrm{OH}, \mathrm{NH}, \mathrm{C}_{3}, \mathrm{C}_{2}, \mathrm{CH}$, and $\mathrm{NH}_{2}$ molecules were observed on at least one occasion. We converted the observed molecular column densities to production rates using a Haser (1957) model. We defined a restricted data set of comets that had at least 3 nights of observations. The restricted data set consists of 59 comets. We used ratios of production rates to study the trends in the data. We find two classes of comets: typical and carbon-chain depleted comets. Using a very strict definition of depleted comets, requiring $\mathrm{C}_{2}$ and $\mathrm{C}_{3}$ to both be depleted, we find $9 \%$ of our restricted data set comets to be depleted. Using a more relaxed definition that requires only $\mathrm{C}_{2}$ to be below a threshold (similar to other researchers), we find $25 \%$ of the comets are depleted. Two-thirds of the depleted comets are Jupiter Family comets, while one-third are Long Period comets. 37\% of the Jupiter Family comets are depleted, while $18.5 \%$ of the Long Period comets are depleted. We compare our results with other studies and find good agreement.
\end{abstract}

Keywords: Comets, composition, Spectroscopy

\section{Introduction}

Since the time that the first spectra of comets were obtained, it has been remarked that all cometary spectra are very similar, with the most

*Corresponding Author

Email addresses: anita@barolo.as.utexas.edu (A. L. Cochran), ebarker40@comcast.net (E. S. Barker), candaceg@nmsu.edu (C. L. Gray) marked difference being the amount of continuum present. This simple observation leads to the question of whether all comets share the same composition or if there are comets with fundamentally different compositions. If differences are seen, then an additional question of the origin of the differences would be raised: are differences the result of different formational scenarios or different evolutionary scenarios? These 
questions are not just curiosities. We believe that comets are leftovers from the formation of the Solar System, that they have undergone only small changes since their time of formation, and that they represent the building blocks of the jovian planets. Thus, understanding their chemistry is important for our understanding of the conditions at the start of the formation of the Solar System.

With these questions in mind, we began a program in 1980 to obtain spectra of comets using low resolution spectrographs at McDonald Observatory. This program continued through 2008. We obtained observations of 132 different comets, some at more than one apparition. This paper reports on the observations of 130 of those comets. 29P/Schwassmann-Wachmann 1 is left out because its almost-circular, $5 \mathrm{AU}$ orbit and random outbursts make it an unusual comet that has been described in several previous papers (Cochran et al. 1980, 1982, 1991a, 1991b; Cochran and White 1993). D/Shoemaker-Levy 9 broke into many pieces prior to impacting Jupiter in 1994. Observations of the pieces had to be obtained in a different manner from the other comets and these are detailed in Cochran et al. (1994).

Some of the comets described in this present paper were already discussed in prior papers (e.g. Cochran et al. 1987, 1989, 1992, 2009; Cochran and Barker 1987). They are included again for completeness so that all the observations can be found in the same place. In addition, all observations were analyzed in a consistent manner in this paper. Thus, this paper supersedes its predecessors.

This is not the only survey of its kind to be carried out. A'Hearn et al. (1995, hereafter AH95) reported on observations of photometry of 85 comets. Fink (2009, hereafter
F09) presented spectroscopic observations of 92 comets. Langlund-Shula and Smith (2011, hereafter LS11) observed 26 comets. We will compare our results with these studies near the end of this paper.

\section{Observations}

The observations described in this paper were obtained with three different instruments on two telescopes at McDonald Observatory. The vast majority of the observations were obtained with two spectrographs at the Cassegrain focus of the 2.7m Harlan J. Smith telescope. A few observations were obtained at the Cassegrain focus of the $2.1 \mathrm{~m}$ Otto Struve telescope.

The earliest observations were obtained with the Intensified Dissector Scanner (IDS) spectrograph on the $2.7 \mathrm{~m}$ telescope. This instrument had two $4 \times 4$ arcsec apertures, separated by 52 arcsec along an east/west line, that were imaged on a photocathode. Spectra were obtained by rapidly scanning the complete wavelength range, which was typically $3200-6000 \AA$, at $11 \AA$ resolution. A complete spectral scan took $50 \mathrm{sec}$ so all wavelengths were essentially observed simultaneously. The comet optocenter was alternately imaged through the "A" slit with the "B" slit imaging 52 arcsec away in the coma. Then the telescope was nodded to image the comet in $\mathrm{B}$ and coma in $\mathrm{A}$. The sequence generally went ABBA. Sometimes, the telescope was set so that the comet optocenter was between the slits or at some other known position and spectra were obtained without nodding the telescope. Scans were repeated until sufficient signal/noise was obtained.

The photocathode made the instrument extremely sensitive. Thus, we were able to obtain spectra of comets as faint as $\mathrm{V}=19.5$. However, 
it also made it impossible to observe very bright objects. Thus, our standard stars were limited to those that were fainter than $\mathrm{V}=10$ and were taken from the list of Stone (1977). In addition, we were not able to obtain observations of solar analog stars with this instrument (none are faint enough) and had to use fluxes from the Arvesen et al. (1969) atlas convolved with the slit function of the instrument. The Arvesen et al. atlas is a whole-disk integrated high-resolution atlas suitable for our purposes. It is not the only available atlas and there are known differences in the flux calibration between the Arvesen et al. atlas and other atlases, especially below $4000 \AA$. Thus, the Arvesen et al. atlas might not be a perfect match for the Sun. However, because we color weight the solar spectrum (see discussion below) before we use it, the most critical feature for our purposes is that the lines are correct in wavelength and strength. The Arvesen et al. atlas is quite acceptable for our purposes.

The second instrument to be used was the Large Cassegrain Spectrograph (LCS) at the $2.7 \mathrm{~m}$ telescope. This was a long slit CCD spectrograph with various gratings. The bulk of the observations were obtained with a grating set to cover the spectral range from $3000-5700 \AA$ at $7.5 \AA$ resolution. Occasionally, we tilted the grating further to the red to observe other features or used a higher resolving power grating to study some bands in more detail. The slit was $\sim 140$ arcsec long and could be oriented at any position angle by rotating the instrument on the back of the telescope. Each pixel on the detector covered a spatial interval of 1.28 arcsec. The slit width was variable but was typically set to 2 arcsec for comet observations and 10 arcsec for standard star observations (so that no flux was lost). Generally, solar analog stars were observed at least once per run and often every

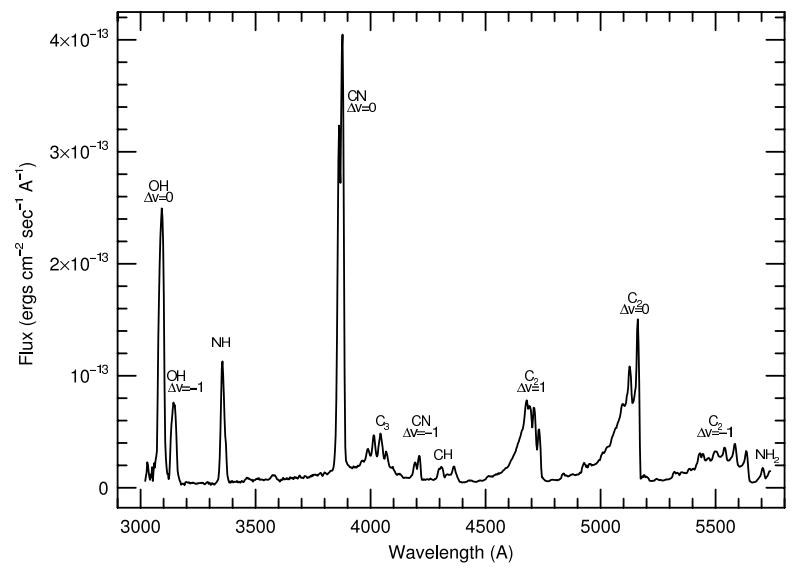

Figure 1: A spectrum of comet $122 \mathrm{P} / \mathrm{deVico}$ obtained with the LCS on 27 September 1995 UT when the comet was at $0.69 \mathrm{Au}$ from the Sun. The various molecular bands in our bandpass are marked on the spectrum.

night. When not observed for whatever reason, convolution of the slit function with the Arvesen et al. (1969) atlas was used for a solar spectrum.

With the LCS, we would always observe with the optocenter imaged on the chip at least once every night. We would then sometimes translate the slit under very accurate telescope control to extend our coverage into the coma. Some nights we also observed at more than one position angle.

The third instrument was the Electronic Spectrograph Number 2 (ES2), used on the $2.1 \mathrm{~m}$ telescope. This instrument is very similar to the LCS and even used the same detectors. The slit position angle could also be changed by rotating the instrument on the back of the telescope. Unlike the LCS, we could not image additional regions of the coma because the $2.1 \mathrm{~m}$ telescope cannot be positioned accurately enough.

Figure 1 shows a spectrum obtained with the LCS of comet $122 \mathrm{P} /$ de Vico on 27 September 1995 UT. This is a very clean spectrum covering 
$1.28 \times 2$ arcsec around the optocenter. The various features observed in our bandpass are noted on the figure. These are all molecular emission bands and are composed of many individual lines that we do not resolve. This comet had a very low dust-to-gas ratio so there is very little continuum to be seen. The $\mathrm{OH}, \mathrm{NH}$ and $\mathrm{CN}$ features tend to be relatively sharp and narrow and are easy to see and measure. The $\mathrm{C}_{3}$ and $\mathrm{C}_{2}$ bands are broad and can easily be lost in noise when weaker, in spectra with lower signal/noise or when the continuum due to sunlight reflecting off the dust is very strong. The latter is a problem because the continuum removal can represent a large source of uncertainty and add noise to the result, even when the signal/noise of the comet spectrum is very high. The continuum decreases faster with cometocentric distance than does the $\mathrm{C}_{2}$ or $\mathrm{C}_{3}$ band strength, so this is more of a problem on the optocenter points than further into the coma. The $\mathrm{CH}$ is always very weak and difficult to measure. In the vast majority of the spectra reported in this paper, our bandpass included the features due to $\mathrm{CN}, \mathrm{C}_{3}, \mathrm{CH}$, and $\mathrm{C}_{2}$. We could never measure $\mathrm{OH}$ and $\mathrm{NH}_{2}$ simultaneously because, as shown in the spectrum, the wavelength separation is beyond the bandpass of the instrument (only part of the $\mathrm{NH}_{2}$ is seen on this spectrum). In addition, high airmass makes observation of the $\mathrm{OH}$ very difficult (though ironically, the de Vico spectrum was obtained when the comet was at 2.5 airmasses and we were able to observe the $\mathrm{OH}$ in this comet even when it was at 3.8 airmasses (!) because the comet was so close to the Sun).

The IDS was used from 1980 through 1989. We started using the LCS in 1985. Thus, there was a period from 1985 - 1989 when we interchanged which instrument was used (for reasons that have long since been forgotten!). The LCS was used with an RCA $512 \times 512$ pixel CCD from 1985 through 1986. This detector did not have UV response so was used in a redder setting but with more limited bandpass because of the smaller number of pixels than the subsequent CCD. From 1988, we used a Texas Instruments $800 \times 800$ pixel CCD that was UV sensitized. We also used the Texas Instrument CCD on ES2. In all cases, the telescope was tracked at the comet's rate of motion on the sky and we guided the telescope directly off the slit. Thus, positional accuracy was generally quite good.

As would be expected with a survey that spanned so many years, we changed our observational philosophy during the course of the survey. When we started, we wanted to be as inclusive as possible and would observe as many comets to $\mathrm{V} \sim 19.5$ as we could. We therefore sometimes observed comets at heliocentric distances greater than 2.5 AU, where they were generally not very active. We sometimes also sacrificed signal/noise for the opportunity to observe another comet. We might only observe a particular comet on a single night.

As our survey progressed and as we changed instruments, we tended to concentrate more on targets that were brighter and at smaller heliocentric distances. We chose opportunities to observe the same comet repeatedly over opportunities to observe additional comets. In addition, comets of special interest (mission targets, close approaches, etc.) began to dominate the survey. Starting in 1995, whenever a comet was bright enough, we would observe at very high spectral resolution instead of with the instruments described here. Those observations are not included in this paper. 


\section{Reduction and Analysis}

The routine reduction of the data is very similar for each of the instruments. First, we removed the bias for the CCDs (the IDS did not have a bias) and flat fielded the data. Since the CCD instruments (LCS and ES2) are long slit instruments, we extracted the stellar spectra using variance weighting (an estimation of the variance created from a noise model based on the gain and readnoise parameters and a smooth profile function) of the slit profile to collapse the stellar spectral image into a $1 \mathrm{D}$ spectrum. The comet spectra for the CCD instruments were left as $2 \mathrm{D}$ spectral images. For all instruments, we observed arc lamps in order to define the wavelength correspondence of each pixel. For the IDS, we used a fifth or sixth order polynomial to fit the dispersion. For the LCS, we needed to fit the wavelength in both the spectral and spatial directions. We used a fifth order polynomial in the spectral direction and a fourth order polynomial in the spatial direction. We would use our spatial fit to transform the spectral image so that the spectra were aligned with rows and the spectrum was no longer curved along the chip. Finally, the cometary and solar analog spectra were flux calibrated with the standard star observations to convert the spectra into flux versus wavelength.

The cometary spectrum consists of an amalgamation of two types of contributions. The coma gas yields a molecular band spectrum in emission, generally from resonance fluorescence. Sunlight reflecting off the dust creates a continuum underlying the gas spectrum. In addition, there is a third spectrum superimposed on the cometary spectrum and that is the spectrum of the Earth's atmosphere (the telluric night sky emission spectrum).
For the purposes of this paper, we are interested in the quantities of the gases that are imaged through our aperture. Thus, we need to remove both the telluric night sky emission spectrum and the solar spectrum. The most prominent telluric feature in our spectrum is the $\mathrm{O}\left({ }^{1} \mathrm{~S}\right)$ feature at $5577 \AA$. The telluric spectrum also has bands in the UV that must be removed. To remove the telluric spectrum, we would use either a dedicated sky observation (always the case for the IDS), obtained well removed from any comet, or we would use the spectrum at the end of the long slit (for the LCS and ES2) if a comet was not very extended and there were no gas features seen in that part of the spectral image. We would weight the sky spectrum by using the strength of the $5577 \AA$ band above the local continuum and then would subtract the sky spectrum from the comet spectrum. Note that, for many comets, there is the $\mathrm{C}_{2} \Delta v=-1$ band in the region of the $5577 \AA$ line so we needed to define the continuum with respect to this band. Because the $5577 \AA$ feature is by far the dominant telluric signal, slight mis-weighting of the telluric spectrum was not terribly critical except for the $\mathrm{C}_{2} \Delta v=-1$ band. We never use this band in our analysis because there could be uncertainties induced in it by this process. We see no other strong telluric features (McDonald Observatory does not suffer from street lighting induced features). There are some weak features seen, including the Herzberg bands of $\mathrm{O}_{2}$, predominantly below $3600 \AA$.

Some comets have very strong continuum spectra because their comae contain a great deal of dust to reflect sunlight; others have very weak continua. To remove the solar spectrum, it would be ideal to have observations of the Sun observed with the same instrument. This is not possible. The next best thing would be to ob- 
serve a star that is a strong match to the properties of the Sun. None of the tabulated solar analogs is fainter than $\mathrm{V}=10$ so we were not able to observe them with the IDS, as noted above. For the LCS and ES2, we could observe solar analogs but considerations such as weather or availability meant that we did not always observe one. The final resort for a solar spectrum was to use a full disk spectrum of the Sun. These are typically observed at high spectral resolution and must be convolved with a slit function to match our instrumental resolution. We adopted the solar spectrum of Arvesen et al. (1969) for this purpose.

It should be noted that the small particles of dust in the coma result in the spectrum of the reflected sunlight being reddened when it is reflected off the dust. Thus, the solar spectrum does not exactly match the continuum we wish to remove. To fix this problem, we measured the flux in up to 11 continuum bandpasses in both the comet and the solar spectrum and weighted the solar spectrum to match the same color. Use of so many continuum regions allows us to match any non-linear reddening that exists. We used a smooth spline function to fit these continuum regions. The resultant weighting curve was never a straight line. This color weighted spectrum was then removed from the comet spectrum. This process would alleviate any issues with the UVblue flux calibration of the Arvesen et al. (1969) atlas or with stars of slightly different colors than the Sun.

In the case of the IDS, each observation would contain spectra of two positions - often the optocenter and 52 arcsec away. Each position was handled separately in the process described above. The off position still included the coma of the comet so was not used as a source of a sky spectrum unless the off spectrum was de- void of any emissions. For the LCS and ES2, the long slit covers $>110$ pixels spatially. For high signal/noise LCS or ES2 spectra, we would treat each spatial pixel as a separate spectrum for the telluric and solar removal. For lower signal/noise observations, we would sometimes bin together pixels in the spatial direction and then apply the removal of the telluric and solar spectra (the concept of binning does not apply to the IDS). Figure 2 shows an example of handling the optocenter pixel for comet d'Arrest on 28 September 1995. The optocenter spectrum has the strongest continuum. The continuum due to the dust weakens faster with spatial distance from the optocenter than does the gas emission.

Once the telluric and solar spectra were removed, we could now measure the quantity of gas in each emission band. First, we fit a continuum to the region around each of the molecular bands of interest, removed the continuum and integrated the flux above the continuum. Though there should be no continuum left after removal of the telluric and solar spectra, in reality, the color weighting is imperfect because there are few true continuum regions for the weighting. The fitted continuum at this point provides a consistent level to integrate above.

The integrated fluxes are then converted to column densities using the standard efficiency factors listed in Table 1, These assume the coma is optically thin. This will always be the case in the outer coma. For a few of the most active comets, the collisional zone might be big enough to affect the inner pixel. However, our pixels are generally much larger than the affected region. One comet for which this would be a problem if it had been observed near $1 \mathrm{AU}$ is comet C/1995 O1 (Hale-Bopp). However, as our observations were at $4 \mathrm{AU}$, this is not a problem for our data.

Note that at this point we generally had col- 
Table 1: Constants for Converting Fluxes to Column Densities

\begin{tabular}{|c|c|c|}
\hline Molecule & $\begin{array}{c}\text { Constant } \\
\left(\mathrm{ergs} \mathrm{mol}^{-1} \mathrm{sec}^{-1}\right)\end{array}$ & $\operatorname{Ref}^{a}$ \\
\hline $\mathrm{OH}$ & $f\left(\dot{R}_{h}\right)$ & 1 \\
\hline $\mathrm{NH}$ & $f\left(\dot{R}_{h}\right)$ & 2 \\
\hline $\mathrm{CN}$ & $f\left(\dot{R}_{h}\right)$ & 3 \\
\hline $\mathrm{C}_{3}$ & 12.42 & 4 \\
\hline $\mathrm{CH}$ & 12.98 & 4 \\
\hline $\mathrm{C}_{2}(\Delta v=1)$ & 12.62 & 5 \\
\hline $\mathrm{C}_{2}(\Delta v=0)$ & 12.35 & 5 \\
\hline $\mathrm{NH}_{2}$ & 13.73 & 6 \\
\hline \multicolumn{3}{|c|}{$\begin{array}{l}\text { For } \mathrm{OH}, \mathrm{NH} \text { and } \mathrm{CN} \\
\qquad \log \mathrm{M}=f\left(\dot{R}_{h}\right)+\log \mathrm{L}+2 \log R_{h} \\
\text { For } \mathrm{C}_{3}, \mathrm{CH}, \mathrm{C}_{2} \text {, and } \mathrm{NH}_{2} \text { : } \\
\qquad \log \mathrm{M}=\text { const }+\log \mathrm{L}+2 \log R_{h} \\
\text { where } \mathrm{M}=\text { column density, } \mathrm{L}=\text { band intensity, } \mathrm{R}_{h} \\
=\text { heliocentric distance }(\mathrm{AU}), \dot{R}_{h}=\text { heliocentric radial } \\
\text { velocity }(\mathrm{km} / \mathrm{sec}), f\left(\dot{R}_{h}\right) \text { is the fluorescence efficiency } \\
\text { as a function of heliocentric distance. }\end{array}$} \\
\hline \multicolumn{3}{|c|}{$\begin{array}{l}{ }^{a} \text { References }-1: \quad g \text {-factor from Schleicher and } \\
\text { A'Hearn 1982; 2: } g \text {-factor from Kim et al. 1989; } 3 \text { : } \\
\text { Tatum and Gillespie (1977); 4: Cochran and Barker } \\
\text { (1985); 5: Oliversen et al. (1985) and } g \text {-factor from } \\
\text { A'Hearn et al. (1985); 6: } g \text {-factor from Tegler and } \\
\text { Wyckoff (1989) }\end{array}$} \\
\hline
\end{tabular}

umn densities for each observed molecule at multiple positions within the coma. For some comets, we might just have an optocenter point, while for others we had many hundreds of data points, covering distances to greater than $10^{5} \mathrm{~km}$ from the optocenter. Figure 3 shows an example of the column densities as a function of position for comet Halley obtained using both the LCS and IDS. We needed a way to be able to intercompare comets that had disparate numbers of observed positions in the coma and a variety of observed molecules. We did this by model- ing the comets using the computationally simple Haser (1957) model. The Haser model is not very physical as it assumes that the cometary comae are spherically symmetric and that all gas only flows outwards from the nucleus. As we will show later, spherical symmetry is often violated. We also expect that dissociation will allow the daughter species to travel in any random direction. Despite these shortcomings, we still chose to use this model because it is an easy way to intercompare comets and to compare with other researchers. We adopted the Haser model 
Table 2: Adopted Haser Model Scale Lengths

\begin{tabular}{|c|c|c|c|}
\hline Molecule & $\begin{array}{c}\text { Parent } \\
\text { Scale length } \\
(\mathrm{km})\end{array}$ & $\begin{array}{l}\text { Daughter } \\
\text { Scale length } \\
(\mathrm{km})\end{array}$ & Reference $^{a}$ \\
\hline $\mathrm{OH}$ & $2.4 \times 10^{4}$ & $1.6 \times 10^{5}$ & 1 \\
\hline $\mathrm{NH}$ & $5.0 \times 10^{4}$ & $1.5 \times 10^{5}$ & 2 \\
\hline $\mathrm{CN}$ & $1.7 \times 10^{4}$ & $3.0 \times 10^{5}$ & 3 \\
\hline $\mathrm{C}_{3}$ & $3.1 \times 10^{3}$ & $1.5 \times 10^{5}$ & 3 \\
\hline $\mathrm{CH}$ & $7.8 \times 10^{4}$ & $4.8 \times 10^{3}$ & 4 \\
\hline $\mathrm{C}_{2}{ }^{b}$ & $2.5 \times 10^{4}$ & $1.2 \times 10^{5}$ & 3 \\
\hline $\mathrm{NH}_{2}$ & $4.1 \times 10^{3}$ & $6.2 \times 10^{4}$ & 5 \\
\hline \multicolumn{4}{|c|}{$\begin{array}{l}{ }^{a} \text { References }-1 \text { : Cochran and Schleicher (1993); 2: } \\
\text { Randall et al. (1992); 3: Cochran (1986); 4: Cochran } \\
\text { and Cochran (1990); 5: Cochran et al. (1992) } \\
{ }^{b} \mathrm{C}_{2} \text { parent scales as } \mathrm{R}_{h}^{2.5} \text {, all others as } \mathrm{R}_{h}^{2}\end{array}$} \\
\hline
\end{tabular}

scale lengths listed in Table 2 for this purpose. These are not necessarily the best fits to all of the data but offer a consistent set of parameters without too many degrees of freedom. Our choice of scale lengths, as compared with other authors, is discussed later. In addition, we use a modified Delsemme (1982) velocity scaling law of $v=0.85 R_{h}^{0.5}$ for the models.

Table 3 is representative of the information that we gathered for each comet. For each comet and each night of observation, we list the observational circumstances, the instrument, the position angle of the slit for the LCS and ES2, measured north through east (left blank for the IDS since the apertures were fixed on an east/west line) and the values of the measured production rates (denoted with a $\mathrm{Q}(\mathrm{X})$ where $\mathrm{X}$ is the species in question). For the production rates, we list the log of the production rate for each molecule along with the binning (for LCS and ES2) and the number of data points that went into the fit. Note that for some comets and some nights we would have more than one spectral image per night, resulting in many more data points than are covered by the slit in a single spectral image. These spectral images were sometimes, but not always, centered on different portions of the coma.

The table here is for only a single comet (23P/Brorsen-Metcalf) to give the reader an idea of the nature of the available data. As it is, this representation of the table must be sliced into three parts to fit the table onto a page for printing. Since there are over 650 lines of table with all of the comets, it is impractical to print all of the values. Instead, all of the data are available on-line in the supplementary materials for this paper.

One of the columns in this table is labeled "Group". This column lists the dynamical type of the comet: JFC = Jupiter Family comet (Tisserand parameter $>2$ ); HTC $=$ Halley Type Comet (Tisserand parameter $<2$ and $\mathrm{P}<200$ years $) ; \mathrm{LPC}=$ Long Period Comet $(\mathrm{P}>200$ 
Table 3: Representative Production Rate Table (Broken into 3 parts)

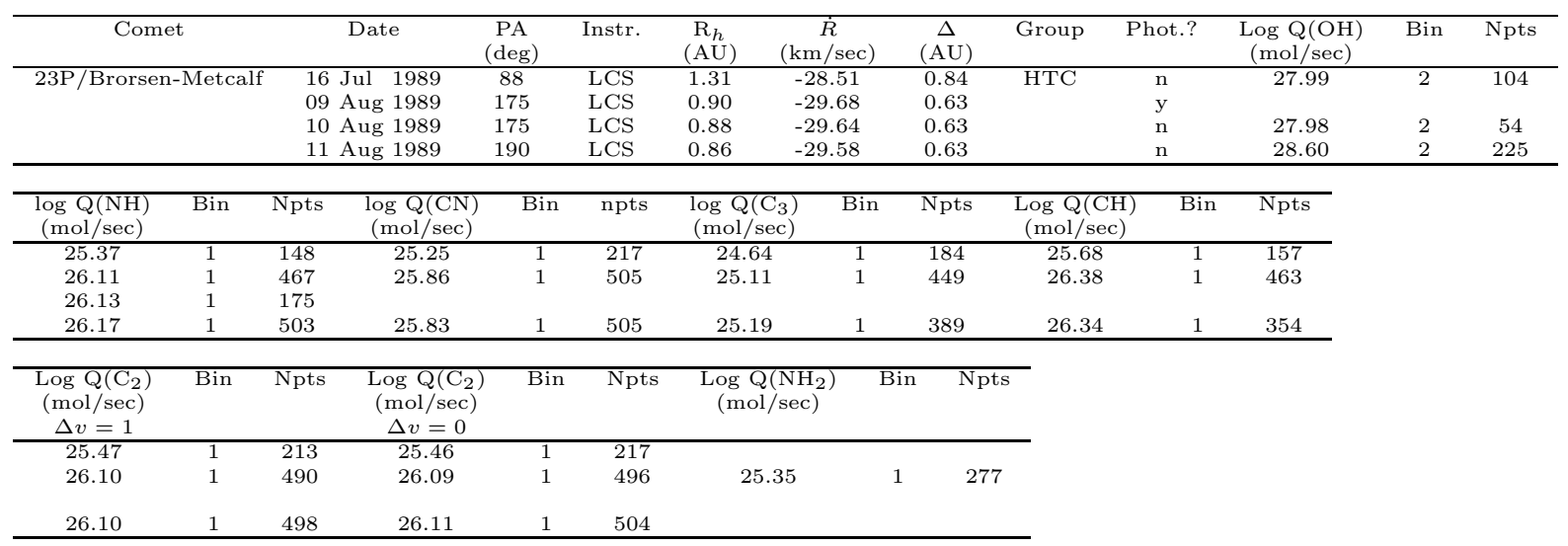

years). The next column is labeled "Phot?". The values for this column are either " $y$ " or " $n$ " to indicate whether or not it was photometric. If it was not photometric, then the production rates are not absolute. However, since all the wavelengths were observed simultaneously, the relative productions of species are still accurate since most clouds are gray (Wing 1967). For the LCS and ES2, we would sometimes obtain multiple spectral images even if not photometric. For purposes of the analysis, we would scale all the column densities (as a group from a single spectral image) to the spectral image that was the brightest. For this scaling, all molecules were scaled by the same amount. That we could use a single scaling factor to bring molecules at disparate wavelengths into good alignment is another indicator that the clouds are generally gray. The resultant production rates are still not absolute but, again, the relative production of species is correct.

When we observed at more than one slit position angle with the LCS or ES2, we would keep the different position angles separate for the analysis. The position angle should not affect the production rates (as long as the optocenter is in each image) so this serves as a check on the derived production rates. The exception to this policy was the Encke observations of October 2003 as we used so many different position angles that it was impractical to keep them separate.

Inspection of Table 3 shows that some of the entries for production rates are blank. In the case of Brorsen-Metcalf, these blanks are because the bandpass did not include some of the molecules on some of the nights. On 16 July 1989 and 11 August 1989, we observed with our nominal setup that covered $\mathrm{OH}$ but not $\mathrm{NH}_{2}$. On, 9 August 1989, we changed the grating angle to observe $\mathrm{NH}_{2}$ but not $\mathrm{OH}$. On 10 August 1989, we employed a higher resolving power grating and only observed $\mathrm{OH}$ and $\mathrm{NH}$.

For other comets, there are blank entries even when we covered a molecule in our setup. This means that we did not detect the molecule, generally because the data were too noisy. We consider that we should be able to measure CN, $\mathrm{C}_{3}$, and $\mathrm{C}_{2}$ in all comets $(\mathrm{OH}$ and $\mathrm{NH}$ are affected by airmass and low detector response, $\mathrm{CH}$ 


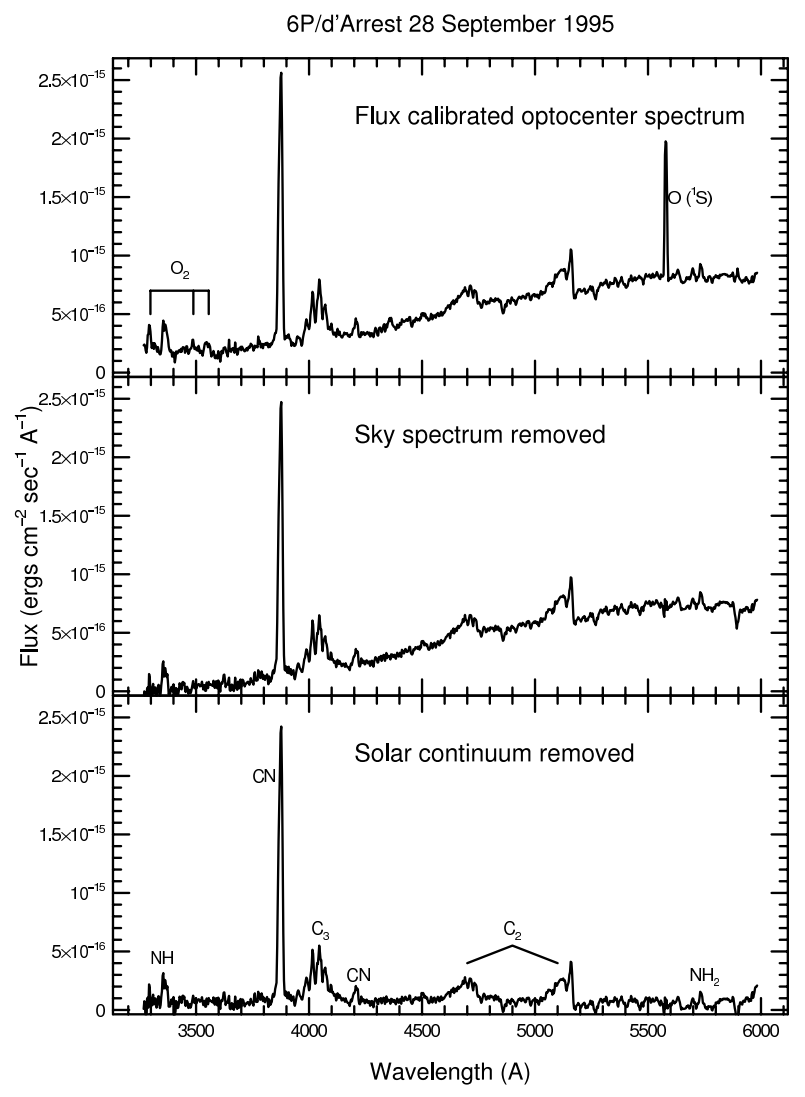

Figure 2: This figure demonstrates the process of removal of the telluric night sky emission and solar spectra from the original spectrum of comet $6 \mathrm{P} / \mathrm{d}$ 'Arrest. The top panel shows the flux and wavelength calibrated spectrum of the comet before removal of either of these spectra. Note the strong feature at $5577 \AA$. This is the $\mathrm{O}\left({ }^{1} \mathrm{~S}\right)$ band in the telluric spectrum. The $\mathrm{O}_{2}$ Herzberg bands are also marked. The continuum results from sunlight reflecting off the dust. The middle panel shows the same spectrum but with the telluric spectrum removed using an independent observation of the sky. The bottom panel shows that spectrum after the removal of the solar continuum. In this case, the spectrum of solar analog Hyades 64 was removed after weighting the continuum to match the colors of the star and the dust (see text).

is very weak and $\mathrm{NH}_{2}$ is often off the chip). We can put upper limits on the column den-

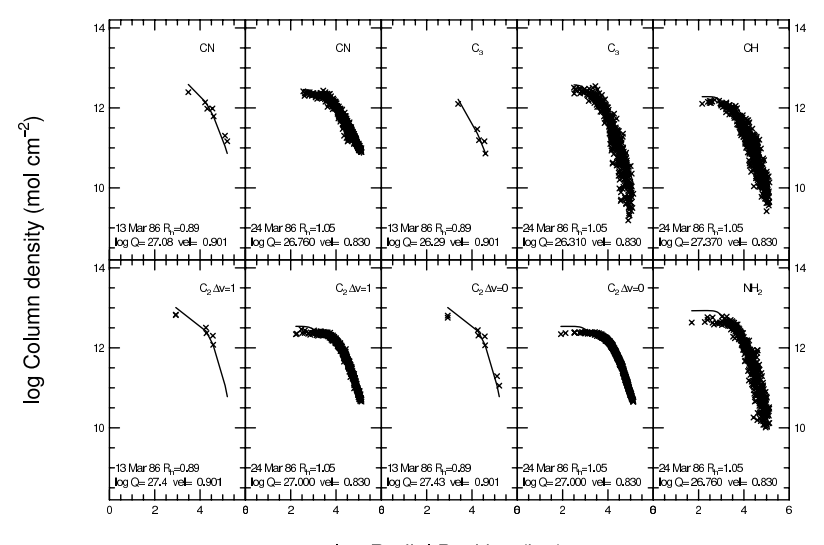

Figure 3: The column densities as a function of position in the coma are shown for comet Halley. In this figure, we show the data sets from both the IDS (13 March 1986) and LCS (24 March 1986). For the IDS, we only observed 4 bands, while we observed 6 bands with the LCS. Note the much larger number of data points obtained with the LCS, the result of the long slit. Superimposed on the data are the Haser model fits to the data. The production rates, $\log \mathrm{Q}$, are in $\mathrm{mol} \mathrm{sec}^{-1}$; the velocity, vel, is in $\mathrm{km}$ $\mathrm{sec}^{-1}$; the heliocentric distance, $\mathrm{R}_{h}$, in in $\mathrm{AU}$.

sities of these three molecules by looking at how the data in the bandpass of the emission band deviates from the continuum. To measure an upper limit, we first fit a continuum to the first and last three points in the bandpass. Next, we computed a standard deviation, $\sigma=\operatorname{sqrt}\left[\Sigma(\text { observed }- \text { continuum })^{2} /(N-1)\right]$, where $N$ is the number of data points in the bandpass. The upper limit is then defined as U.L. $=0.75 \sigma B$, where $B=$ the bandpass in $\AA$. This is a $3 \sigma$ upper limit.

Figure 4 shows an example of a comet for which we detected the $\mathrm{CN}$ band but there is no obvious $\mathrm{C}_{3}$ or $\mathrm{C}_{2}$. This spectrum, of comet $192 \mathrm{P} /$ Shoemaker-Levy at $\mathrm{R}_{h}$ of $1.84 \mathrm{AU}$, has had the telluric and solar spectra removed. The bandpasses for the $\mathrm{C}_{3}$ and $\mathrm{C}_{2} \Delta v=0$ bands are marked on the figure. Included are the measured 


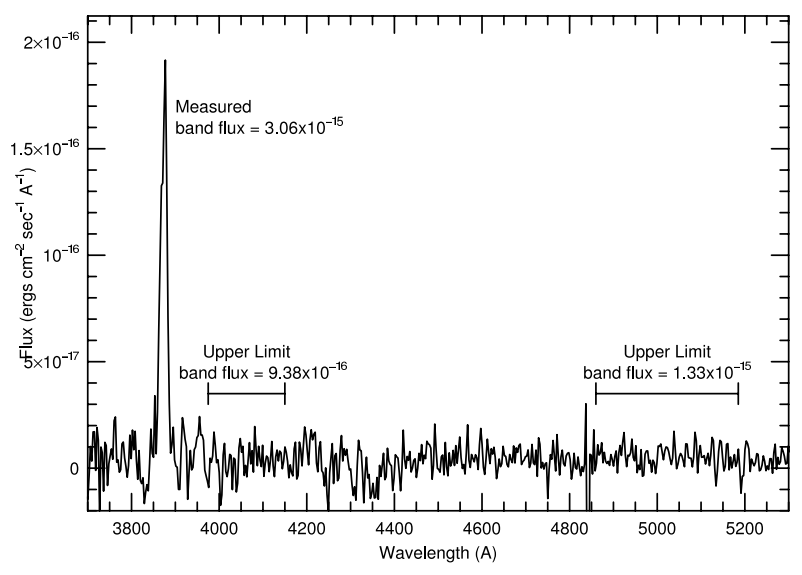

Figure 4: A spectrum of comet 192P/Shoemaker-Levy is shown. $\mathrm{CN}$ is obviously visible but there is no sign of the $\mathrm{C}_{3}$ or $\mathrm{C}_{2}$. By measuring the noise in the bandpass where we would expect to detect these features, we can derive upper limits for the amount of gas that could be attributable to these species. These upper limits are $3 \sigma$ limits as discussed in the text.

integrated band intensity for $\mathrm{CN}$ along with the $3 \sigma$ upper limit integrated fluxes for $\mathrm{C}_{3}$ and $\mathrm{C}_{2}$. In this example, it should be obvious that there cannot be much $\mathrm{C}_{2}$ or $\mathrm{C}_{3}$ hidden in this spectrum.

In general, the upper limits are not very constraining. If we observed all three of $\mathrm{CN}, \mathrm{C}_{3}$, and $\mathrm{C}_{2}$ on at least one of the nights we observed a particular comet, we did not tabulate the upper limits for other nights because we believed that the actual detections were more meaningful (the upper limit measurements are much more labor intensive than the usual column density measurements because we never developed the same degree of automation in their computation that we have for column densities). However, we have included upper limits for the cases when $\mathrm{CN}$ was detected but $\mathrm{C}_{2}$ and $\mathrm{C}_{3}$ were not both detected. We have only one case, comet $\mathrm{C} / 1984 \mathrm{~K} 1$ (Shoemaker), when we detected $\mathrm{CN}$ and $\mathrm{C}_{3}$ but not $\mathrm{C}_{2}$ and one case, $\mathrm{C} / 1992 \mathrm{~W} 1$ (Oshita), where we observed $\mathrm{CN}$ and $\mathrm{C}_{2}$ but not $\mathrm{C}_{3}$.

Of our sample of 130 comets, there are 20 comets for which we observed no molecules, not even CN. These are tabulated in Table 4, where we list the date, observing circumstances, group and the upper limits for the $\mathrm{CN}$ production rates. We do not include any other upper limits since ratios of upper limits are not at all meaningful. Inspection of Table 4 shows that many of these comets were observed at large heliocentric distance $(>2.5 \mathrm{AU})$, where they would not be expected to produce much emission. However, there are a few examples (108P/Ciffreo, 96P/Machholz 1, 7P/PonsWinnecke, and $125 \mathrm{P} /$ Spacewatch) with observations at $\leq 1.75 \mathrm{AU}$, where we would have expected to detect at least $\mathrm{CN}$. These comets must be very low producers, indeed.

Before we can compare comets, it is important to understand the uncertainties in our measurements. As with all data, these include both systematic and random errors. One source of the systematic errors is our knowledge of the $g$ factors. We differ from A'Hearn et al. (1995) for the $\mathrm{C}_{3}$ and $\mathrm{CN} g$-factors. The different $g$-factors will make it hard to intercompare with other observers but should not affect intercomparisons of comets within a single data set.

For $\mathrm{C}_{3}$, no good oscillator strengths have been measured in the laboratory since $\mathrm{C}_{3}$ is not a stable molecule. Jorgensen et al.. (1989) reviewed the laboratory measurements and found a range in measured oscillator strengths of a factor of 6. We long ago adopted a value of $f=0.016$ (Clegg and Lambert 1982) while A'Hearn et al. use 0.001. Roueff et al. (2002) adopted a value of $f=0.0146$ whereas Ádámkovics et al. (2003) adopted $f=0.016$, in agreement with our value. Our value is consistent with the current liter- 
Table 4: Comets With No Molecules Detected

\begin{tabular}{|c|c|c|c|c|c|c|c|}
\hline Comet & Date & Instr. & $\begin{array}{c}\mathrm{R}_{h} \\
(\mathrm{AU})\end{array}$ & $\begin{array}{c}\dot{R} \\
\mathrm{~km} / \mathrm{sec}\end{array}$ & $\begin{array}{c}\Delta \\
(\mathrm{AU})\end{array}$ & Group & $\begin{array}{c}\text { Log } \mathrm{Q}(\mathrm{CN}) \\
\text { Upper Limit } \\
(\mathrm{mol} / \mathrm{sec})\end{array}$ \\
\hline \multirow[t]{2}{*}{$\overline{47 \mathrm{P} / \text { Ashbrook-Jackson }}$} & 16 Oct 1985 & IDS & 2.41 & -3.52 & 2.16 & JFC & $<24.04$ \\
\hline & 16 Nov 1985 & IDS & 2.36 & -2.54 & 2.47 & & $<24.07$ \\
\hline \multirow[t]{23}{*}{ C/1980 E1 (Bowell) } & 12 Dec 1980 & IDS & 5.38 & -11.85 & 5.56 & LPC & $<25.64$ \\
\hline & 15 Dec 1980 & IDS & 5.36 & -11.83 & 5.49 & & $<25.29$ \\
\hline & 03 Jan 1981 & IDS & 5.23 & -11.65 & 5.07 & & $<25.19$ \\
\hline & 07 Feb 1981 & IDS & 5.00 & -11.29 & 4.33 & & $<25.71$ \\
\hline & 11 Feb 1981 & IDS & 4.98 & -11.25 & 4.26 & & $<25.56$ \\
\hline & 07 Jun 1981 & IDS & 4.27 & -9.72 & 3.88 & & $<24.91$ \\
\hline & 24 Jan 1982 & IDS & 3.39 & -2.24 & 3.92 & & $<25.67$ \\
\hline & 25 Jan 1982 & IDS & 3.39 & -2.19 & 3.90 & & $<25.41$ \\
\hline & 20 Apr 1982 & IDS & 3.38 & 1.84 & 2.82 & & $<24.90$ \\
\hline & 18 May 1982 & IDS & 3.42 & 3.12 & 2.57 & & $<25.25$ \\
\hline & 26 Jun 1982 & IDS & 3.51 & 4.77 & 2.50 & & $<25.40$ \\
\hline & 16 Jul 1982 & IDS & 3.57 & 5.55 & 2.61 & & $<25.21$ \\
\hline & 17 Jul 1982 & IDS & 3.58 & 5.59 & 2.62 & & $<25.17$ \\
\hline & 18 Jul 1982 & IDS & 3.58 & 5.63 & 2.63 & & $<25.11$ \\
\hline & 17 Aug 1982 & IDS & 3.69 & 6.68 & 2.99 & & $<25.14$ \\
\hline & 15 Sep 1982 & IDS & 3.81 & 7.58 & 3.47 & & $<25.15$ \\
\hline & 13 Oct 1982 & IDS & 3.94 & 8.33 & 3.99 & & $<25.06$ \\
\hline & 10 Jun 1983 & IDS & 5.36 & 11.50 & 5.01 & & $<25.33$ \\
\hline & 13 Jun 1983 & IDS & 5.38 & 11.52 & 4.99 & & $<25.08$ \\
\hline & 07 Jul 1983 & IDS & 5.55 & 11.63 & 4.83 & & $<25.30$ \\
\hline & 05 Aug 1983 & IDS & 5.74 & 11.73 & 4.78 & & $<25.07$ \\
\hline & 10 Sep 1983 & IDS & 5.99 & 11.83 & 5.02 & & $<25.10$ \\
\hline & 05 Oct 1983 & IDS & 6.16 & 11.88 & 5.41 & & $<25.11$ \\
\hline \multirow[t]{2}{*}{ C/1981 H1 (Bus) } & 06 Jun 1981 & IDS & 2.53 & -4.46 & 1.91 & LPC & $<23.73$ \\
\hline & 07 Jun 1981 & IDS & 2.52 & -4.39 & 1.92 & & $<23.63$ \\
\hline
\end{tabular}


Table 4: Comets With No Molecules Detected (cont.)

\begin{tabular}{|c|c|c|c|c|c|c|c|}
\hline Comet & Date & Instr. & $\begin{array}{c}\mathrm{R}_{h} \\
(\mathrm{AU})\end{array}$ & $\begin{array}{c}\dot{R} \\
\mathrm{~km} / \mathrm{sec})\end{array}$ & $\begin{array}{c}\Delta \\
(\mathrm{AU})\end{array}$ & Group & $\begin{array}{c}\text { Log } \mathrm{Q}(\mathrm{CN}) \\
\text { Upper Limit } \\
(\mathrm{mol} / \mathrm{sec})\end{array}$ \\
\hline 108P/Ciffreo & 12 Dec 1985 & IDS & 1.75 & 3.94 & 0.79 & JFC & $<23.32$ \\
\hline 57P/duToit-Neujmin-Delporte & 01 Jul 1989 & LCS & 1.98 & -7.46 & 1.19 & JFC & $<23.39$ \\
\hline C/1983 N1 (IRAS) & 02 Apr 1984 & IDS & 4.24 & 13.40 & 3.27 & LPC & $<24.49$ \\
\hline 48P/Johnson & $\begin{array}{l}\text { 08 Jul } 1983 \\
\text { 06 Aug } 1983 \\
\text { 07 Aug } 1983\end{array}$ & $\begin{array}{l}\text { IDS } \\
\text { IDS } \\
\text { IDS }\end{array}$ & $\begin{array}{l}2.51 \\
2.44 \\
2.44\end{array}$ & $\begin{array}{l}-4.45 \\
-3.78 \\
-3.76\end{array}$ & $\begin{array}{l}1.50 \\
1.58 \\
1.59\end{array}$ & JFC & $\begin{array}{l}<23.61 \\
<23.80 \\
<23.21\end{array}$ \\
\hline C/1999 S4 (LINEAR) & $\begin{array}{l}25 \text { Jan } 2000 \\
26 \text { Jan } 2000\end{array}$ & $\begin{array}{l}\text { LCS } \\
\text { LCS }\end{array}$ & $\begin{array}{l}2.97 \\
2.96\end{array}$ & $\begin{array}{l}-21.07 \\
-21.10\end{array}$ & $\begin{array}{l}2.80 \\
2.81\end{array}$ & LPC & $\begin{array}{l}<24.97 \\
<24.45\end{array}$ \\
\hline 77P/Longmore & $\begin{array}{l}\text { 04 Jan } 1981 \\
\text { 17 Apr } 1988\end{array}$ & $\begin{array}{l}\text { IDS } \\
\text { IDS }\end{array}$ & $\begin{array}{l}2.98 \\
2.65\end{array}$ & $\begin{array}{l}-5.53 \\
-4.39\end{array}$ & $\begin{array}{l}2.31 \\
2.09\end{array}$ & JFC & $\begin{array}{l}<23.17 \\
<23.23\end{array}$ \\
\hline 96P/Machholz 1 & $\begin{array}{l}\text { 13 Jun } 2007 \\
14 \text { Jun } 2007\end{array}$ & $\begin{array}{l}\text { LCS } \\
\text { LCS }\end{array}$ & $\begin{array}{l}1.62 \\
1.64\end{array}$ & $\begin{array}{l}26.78 \\
26.62\end{array}$ & $\begin{array}{l}0.67 \\
0.68\end{array}$ & JFC & $\begin{array}{l}<23.68 \\
<23.43\end{array}$ \\
\hline 7P/Pons-Winnecke & $\begin{array}{l}06 \text { Apr } 1989 \\
\text { 09 May } 1989\end{array}$ & $\begin{array}{l}\text { IDS } \\
\text { IDS }\end{array}$ & $\begin{array}{l}1.98 \\
1.73\end{array}$ & $\begin{array}{l}-13.12 \\
-12.56\end{array}$ & $\begin{array}{l}1.19 \\
1.17\end{array}$ & JFC & $\begin{array}{l}<23.55 \\
<23.76\end{array}$ \\
\hline 30P/Reinmuth 1 & $\begin{array}{l}18 \text { Nov } 1987 \\
19 \text { Nov } 1987 \\
\text { 24 Dec } 1987\end{array}$ & $\begin{array}{l}\text { IDS } \\
\text { IDS } \\
\text { IDS }\end{array}$ & $\begin{array}{l}2.38 \\
2.38 \\
2.22\end{array}$ & $\begin{array}{l}-8.35 \\
-8.34 \\
-7.57\end{array}$ & $\begin{array}{l}1.43 \\
1.42 \\
1.31\end{array}$ & JFC & $\begin{array}{l}<24.26 \\
<23.81 \\
<24.42\end{array}$ \\
\hline 94P/Russell 4 & 04 Jun 1984 & IDS & 2.38 & 5.24 & 1.70 & JFC & $<24.25$ \\
\hline C/1983 R1 (Shoemaker) & $\begin{array}{l}05 \text { Oct } 1983 \\
06 \text { Dec } 1983\end{array}$ & $\begin{array}{l}\text { IDS } \\
\text { IDS }\end{array}$ & $\begin{array}{l}3.38 \\
3.35\end{array}$ & $\begin{array}{c}-2.25 \\
0.56\end{array}$ & $\begin{array}{l}2.45 \\
3.46\end{array}$ & LPC & $\begin{array}{l}<24.39 \\
<24.48\end{array}$ \\
\hline
\end{tabular}


Table 4; Comets With No Molecules Detected (cont.)

\begin{tabular}{|c|c|c|c|c|c|c|c|}
\hline Comet & Date & Instr. & $\begin{array}{l}\mathrm{R}_{h} \\
(\mathrm{AU})\end{array}$ & $\begin{array}{c}\dot{R} \\
(\mathrm{~km} / \mathrm{sec})\end{array}$ & $\begin{array}{c}\Delta \\
(\mathrm{AU})\end{array}$ & Group & $\begin{array}{c}\log \mathrm{Q}(\mathrm{CN}) \\
\text { Upper Limit } \\
(\mathrm{mol} / \mathrm{sec})\end{array}$ \\
\hline \multirow[t]{4}{*}{ C/1989 A5 (Shoemaker) } & 07 Feb 1989 & IDS & 2.65 & -1.39 & 1.70 & $\mathrm{LPC}$ & $<25.17$ \\
\hline & 08 Feb 1989 & IDS & 2.65 & -1.32 & 1.70 & & $<24.70$ \\
\hline & 04 Apr 1989 & IDS & 2.67 & 2.66 & 2.43 & & $<24.04$ \\
\hline & 08 May 1989 & IDS & 2.74 & 4.93 & 3.00 & & $<24.68$ \\
\hline \multirow[t]{2}{*}{ 128P/Shoemaker-Holt 1} & 18 Nov 1987 & IDS & 3.22 & -2.96 & 2.40 & JFC & $<24.58$ \\
\hline & 11 Dec 1988 & IDS & 3.25 & 3.15 & 2.42 & & $<24.43$ \\
\hline 121P/Shoemaker-Holt 2 & 05 Apr 1989 & IDS & 3.01 & 4.56 & 2.27 & JFC & $<24.15$ \\
\hline \multirow[t]{8}{*}{ 56P/Slaughter-Burnham } & 25 Aug 1981 & IDS & 2.63 & -3.25 & 1.87 & JFC & $<23.30$ \\
\hline & 26 Aug 1981 & IDS & 2.63 & -3.22 & 1.86 & & $<23.36$ \\
\hline & 28 Aug 1981 & IDS & 2.62 & -3.15 & 1.84 & & $<23.56$ \\
\hline & 27 Sep 1981 & IDS & 2.58 & -2.07 & 1.61 & & $<23.50$ \\
\hline & 28 Sep 1981 & IDS & 2.58 & -2.04 & 1.60 & & $<23.47$ \\
\hline & 24 Oct 1981 & IDS & 2.55 & -1.03 & 1.58 & & $<23.81$ \\
\hline & 23 Jan 1982 & IDS & 2.59 & 2.54 & 2.51 & & $<23.81$ \\
\hline & 25 Jan 1982 & IDS & 2.60 & 2.58 & 2.52 & & $<23.45$ \\
\hline \multirow[t]{8}{*}{ 74P/Smirnova-Chernykh } & 26 Oct 1981 & IDS & 4.34 & -1.98 & 3.44 & JFC & $<24.27$ \\
\hline & 13 Oct 1982 & IDS & 3.91 & -2.07 & 3.79 & & $<24.32$ \\
\hline & 14 Oct 1982 & IDS & 3.91 & -2.07 & 3.78 & & $<23.98$ \\
\hline & 16 Dec 1982 & IDS & 3.84 & -1.95 & 2.92 & & $<24.03$ \\
\hline & 06 Dec 1983 & IDS & 3.57 & -0.45 & 3.58 & & $<24.21$ \\
\hline & 08 Dec 1983 & IDS & 3.57 & -0.44 & 3.55 & & $<24.14$ \\
\hline & 25 Feb 1984 & IDS & 3.56 & 0.02 & 2.61 & & $<23.93$ \\
\hline & 31 Mar 1984 & IDS & 3.56 & 0.23 & 2.62 & & $<24.46$ \\
\hline $125 \mathrm{P} /$ Spacewatch & 15 Jun 1996 & LCS & 1.57 & -3.12 & 1.08 & JFC & $<23.65$ \\
\hline
\end{tabular}


ature. However, the oscillator strength is, in essence, a scaling factor for the column densities, so our $\mathrm{C}_{3}$ column densities would be different by a constant factor from those computed with a different oscillator strength.

$\mathrm{CN}$ is a bit more complicated than $\mathrm{C}_{3}$ because one needs to account for the Swings effect. Thus, the fluorescence efficiency is a function of the heliocentric radial velocity of the comet. We have adopted the older Tatum and Gillespie (1977) calculation of the fluorescence efficiency while A'Hearn et al. use a formulation that factors in the heliocentric distance of the comet. Recent calculations by Schleicher (2010) are more complete, covering more heliocentric distances than previous calculations. Schleicher concludes that the values at a particular heliocentric distance vary by about a factor of two with velocity but that the trend of the fluorescence efficiency with radial velocity at different heliocentric distances can be radically different. Schleicher's calculations were published after we had completed the reduction and analysis of much of our data. We did not switch fluorescence efficiencies because of the large investment in manpower at that point. The effect of using the older calculation is not a simple constant offset, as with the $\mathrm{C}_{3}$ oscillator strength. It will be less than the full range of variation for the fluorescence efficiency, or a factor of 2 ( 0.3 in the $\log$ ), and will be responsible for increasing the error bars when values are averaged later in this paper.

Another source of systematic errors is our knowledge of the scale lengths for the model. We do not take into account any change in the strength of the solar UV spectrum nor do we take into account any multi-step dissociation processes. Inspection of the model fits in Figure 3 show examples of good fits $(\mathrm{CN}$ and $\left.\mathrm{C}_{3}\right)$ and poor fits $\left(\mathrm{C}_{2}\right.$ and $\left.\mathrm{NH}_{2}\right)$. The $\mathrm{C}_{2}$ is al- most always a bad fit in the very inner coma because $\mathrm{C}_{2}$ is probably a granddaughter product of its source and the Haser model does not account for the two-step production. In addition, some of these scale lengths work for some, but not all, comets. This problem was noted by LS11 who chose to fit the scale lengths for each comet. We do not do this because then there are too many free parameters in the intercomparison of comets. Indeed, if the UV insolation does not change, the scale lengths are, to first approximation, determined entirely by the solar flux and the distance of the object from the Sun. While collisions might affect the inner coma, the vast majority of the coma is unaffected. Our fits rarely end up being dependent on the inner coma data points, anyway, since we have so many data points at larger cometocentric distances. We described the process of defining the scale lengths in Cochran (1986) and use these or updated scale lengths uniformly for all of our observations. These scale lengths were defined using observations from five comets observed from 0.74 to $1.81 \mathrm{AU}$. We include an expanded discussion of the scale lengths later when we intercompare different data sets.

Probably the most important source of systematic error is the Haser model itself. This model assumes that the cometary coma is spherically symmetric and that gas can only flow outwards. With our long slit observations, we observe that spherical symmetry is rarely a good approximation. The effect of using the Haser model is, in affect, to azimuthally average the coma observations and assume this is a reasonable proxy for the production. Figure 5 is an (albeit extreme) example of the types of asymmetry we observe. In these observations of comet 2P/Encke, obtained on 24 October 2003, we rotated the slit to 7 different position angles. Since 


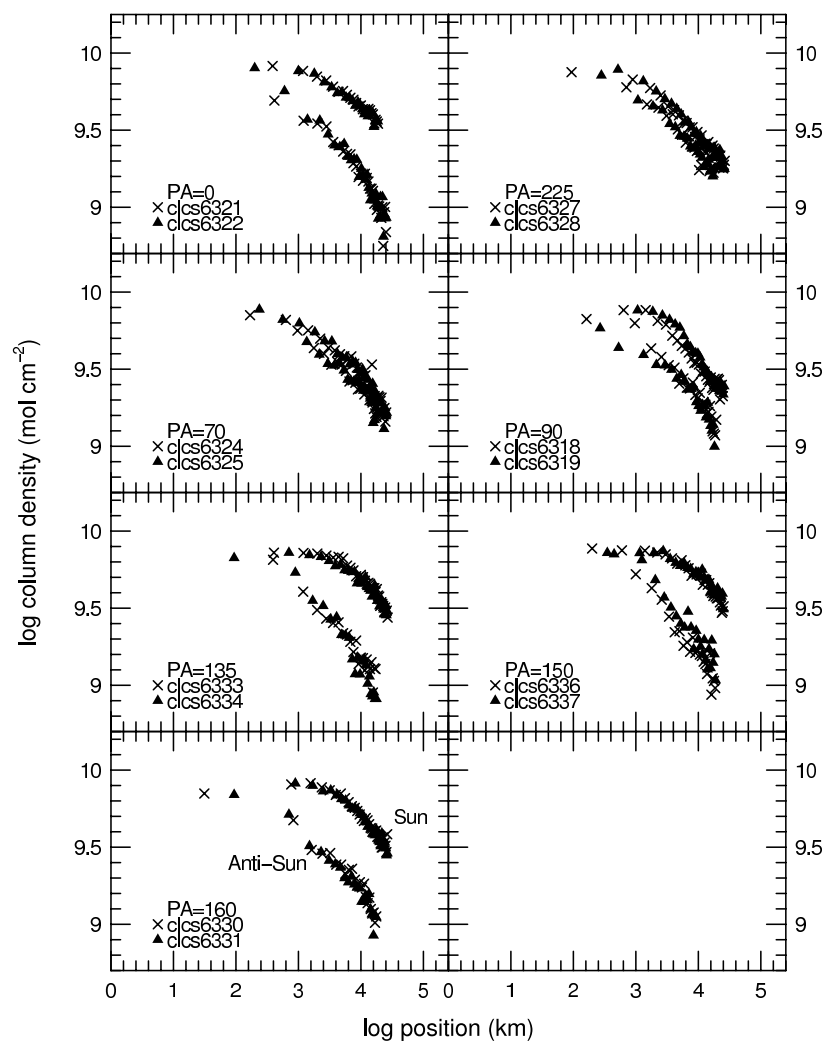

Figure 5: The CN column densities for 2P/Encke observed on 24 October 2003 are shown. Each panel represents a different slit position angle (and that position angle $\pm 180^{\circ}$ on the other side of the optocenter). There are two spectral images at each position angle. Clearly, comet Encke's coma is not symmetric.

the comet was somewhere near the center of the detector, spatially, we actually obtained data on two sides of the optocenter (the listed position angle and the position angle $\pm 180^{\circ}$ ) for each listed position angle. Additionally, at each position angle we obtained two spectral images (that are shown with different symbols in each panel). Inspection of this figure shows that Encke's coma is far from symmetric! The asymmetries observed are not due to clouds or other errors. This is demonstrated by the fact that both spectral images at a single position angle are in excellent agreement (no scaling was done). At some position angles (e.g. $70^{\circ}$, when the slit was positioned perpendicular to the Sun/tail line), the coma looks reasonably symmetric, while at others (e.g. $160^{\circ}$, along the Sun/tail line), there is a factor of 10 difference in column density by the time we are a few thousand $\mathrm{km}$ from the optocenter. A simple model like the Haser model cannot do these data justice. A more complicated model, such as proposed by Ihalawela et al. (2011), allows for deviations from spherical symmetry. However, it should be noted that if we independently fit the different position angles in these Encke data with the Haser model (using both sides of the optocenter together in a single fit), we get the same $\mathrm{CN}$ production rate for each position angle (even though the fits look bad). Asymmetries are observed in many comets. Combi and Fink (1993) showed that Haser model scale lengths could be derived to accommodate time variable asymmetries in comet Halley.

Another source of potential systematic error is the flux calibration. The fluxes of the standard stars are probably only good to $5-10 \%$. In addition, though we used a large aperture for observations of the standard stars, there could be a loss of light from the aperture on some nights. This would cause a decrease in the amount of light we observed for the star and our sensitivity function would then cause a slightly higher flux for the cometary spectra. This would be an extremely rare occurrence, especially for the LCS, since we used a 10 arcsec wide slit.

Clouds would also cause a diminution of the flux. They can affect the comet and standard stars by differing amounts. Thus, it could cause the cometary flux to appear brighter or dimmer. 
As noted above, clouds are gray and we observe all wavelengths at the same time, so we can intercompare the different bands in a ratio sense. In addition, with the long slit, we observe many different positions at the same time, so the column densities as a function of position maintain an accurate relationship. It is only when we were obtaining more than one spectral image that we had to scale the data. As noted above, we would use the same scale factor at all wavelengths.

An additional source of error was continuum removal. This is required in order to study the gas. Sometimes the noise in the continuum meant that the process was not as accurate as we would desire. We mitigate this uncertainty in large part by fitting a continuum under the band prior to computing the integrated band intensity. This corrects for any continuum removal errors.

Finally, an important stochastic error is the photon statistics. For some of our observations, such as the Encke data shown in Figure 5, it is clear that the signal/noise was very high. For others, the signal/noise is not at all good. This is especially true for some of the IDS data where we chose to obtain spectra of another object instead of building signal on the object we were observing. As is normal for stochastic errors in data, we can lower the impact of this noise by collecting more data, i.e. by observing more positions in the coma. For our long slit CCD data, this is a natural consequence of the long slit.

We can judge the affect of the noise on our answer by using a Monte Carlo model to simulate changing the noise. Photon statistical errors affect each point individually. Thus, for a given signal, the measured value could vary based on the signal/noise. To simulate the effect, we took a data set and we changed the value of each data point by selecting randomly from a set of normal

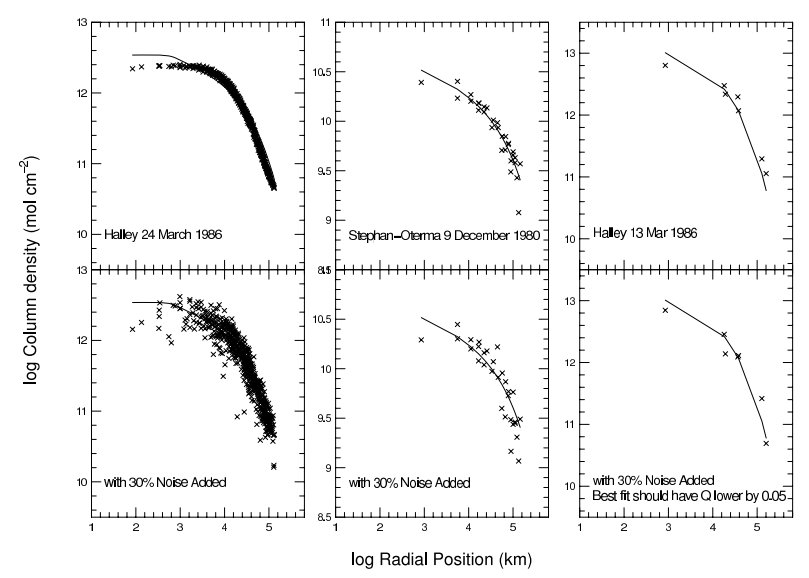

Figure 6: The effects on the column densities due to photon noise are illustrated in these panels. See the text for a discussion of the model. The top panels are three different $\mathrm{C}_{2}$ data sets with 433 individual column densities (Halley 24 March), 30 column densities (Stephan-Oterma 9 December) and only 7 column densities (Halley 13 March). In the top panels, the original data are shown with the best fit Haser model. In the bottom panels, the data are shown after we draw new values from a normalized Gaussian distribution for each point based on $30 \%$ potential error. The model fit is the same one as the real data and looks good for all three data sets. However, while the same model is correct for the Halley 24 March and Stephan-Oterma data, the Halley 13 March data actually required lowering the $\log \mathrm{C}_{2}$ column density by 0.05 . This change in best fit is based on 1000 simulations of noise.

Gaussian deviates. Once each data point was modified, we fit the Haser model and recorded the value for the production rate. We then did this same process repeatedly. Finally, we figured the mean and standard deviations of the derived column densities (in linear space) with the model that was fit to the real data. We tested the errors by running the simulation with 1000 sets of modified data. The range of values that can be selected are generally set by the signal/noise of the data. Figure 6 shows examples of the process in three different $\mathrm{C}_{2}$ data sets - one with 433 data points (Halley from 24 March 1986), 
one with 30 data points (Stephan-Oterma from 9 December 1980) and one with 7 data points (Halley from 13 March 1986). In all three cases, we added $30 \%$ noise, though it is apparent from the scatter in the data that $30 \%$ noise is an over estimate, at least for the 24 March Halley (actual noise about 2.5\%) and for Stephan-Oterma (noise of about 7\%). As shown in the figure, even with the added noise, the Haser model gives the same values for the production rates for those two data sets. For the 13 March Halley data, $30 \%$ error would result in a change of the production rate of only 0.05 in the log. Thus, to the extent that the model and the scale lengths are appropriate, our column densities are very robust when we have more than 25 data points. Fewer data points means that the uncertainty in the production rates might be $5-20 \%$ (stochastically). This is why the number of data points is included in the table that is available on line and shown as an example in Table 3 .

In addition to looking at the number of data points and how much scatter appears in plots of column density versus position, one can check consistency using $\mathrm{C}_{2}$ observations. We measure both the $\mathrm{C}_{2} \Delta v=0$ and $\Delta v=1$ bands in our spectra. The $\Delta v=0$ band has a larger integrated band intensity than the $\Delta v=1$ band. This is accounted for by the different constants used for conversion of the intensities to the column densities. Therefore, the two bands should give independent measurements of the $\mathrm{C}_{2}$ column densities. Figure 7 shows our derived column densities for all comets on all nights on which we measured both bands. There should be a 1:1 correlation. Inspection of Figure 7 shows that in general this is true, though there are some outliers. We use different symbols for the IDS and LCS data. Most of the outliers are the IDS data, as would be expected from the much

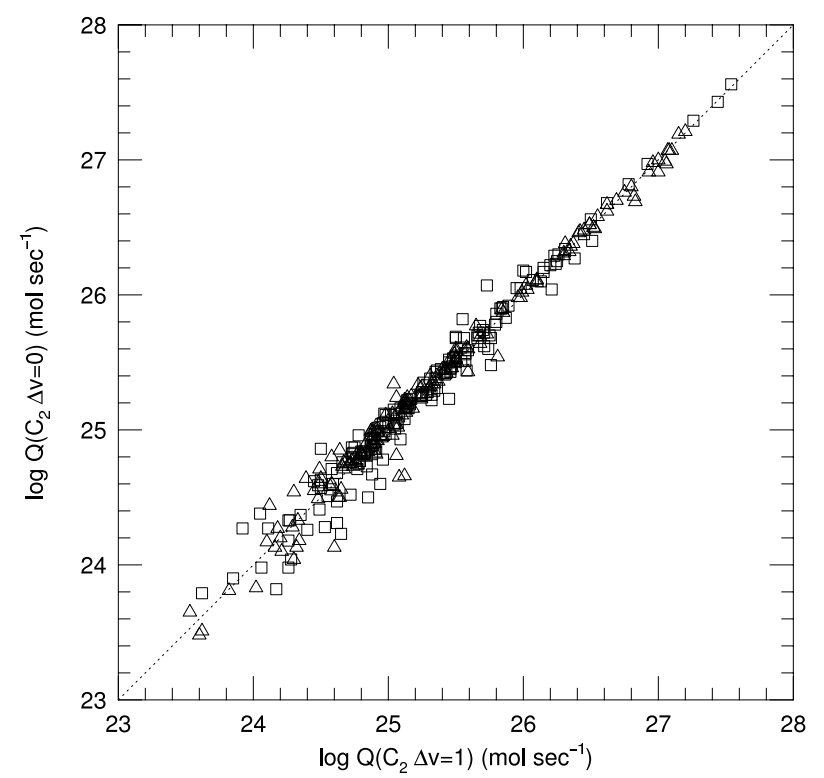

Figure 7: The measured values of the production rates for the $\mathrm{C}_{2} \Delta v=1$ and $\mathrm{C}_{2} \Delta v=0$ bands are shown plotted against one another. We would expect to derive the same production rates from either band. This is what we observe. The IDS data are denoted with squares while the LCS data are denoted with triangles. The uncertainties in the IDS production rates are generally higher than for the LCS production rates since we observe so many more positions with the LCS. The disagreement of the production rates increases at the smallest production rates, indicative of larger uncertainties for weaker comets.

smaller numbers of points obtained for the IDS, resulting in larger error bars on the derived production rates. In particular, we see the greatest deviations from the correlation at the smallest values for the column densities. This is not surprising as the weakest features are the ones with the largest uncertainties. When $\log \mathrm{Q}$ for either band is $>25$, the agreement is generally quite good. A few outliers with $\log \mathrm{Q}>25$ exist, and were checked, but there was nothing to pick one value over the other. 


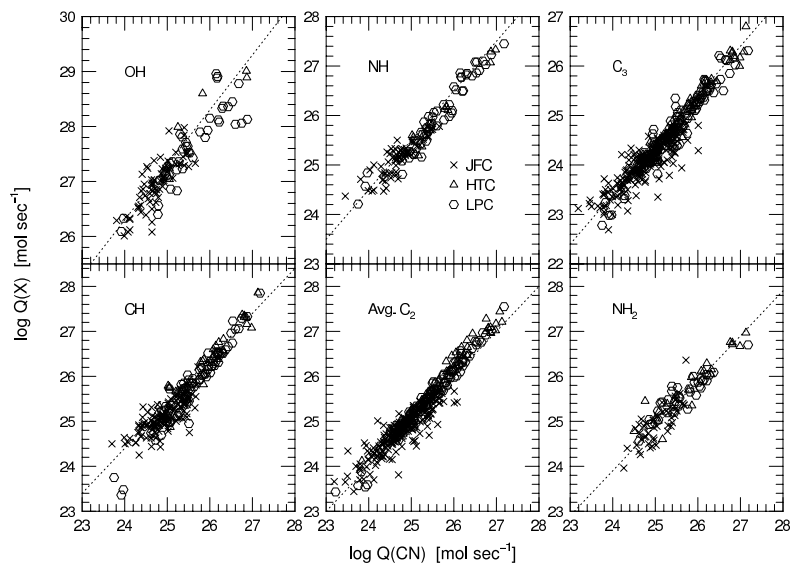

Figure 8: This figure illustrates the observed production rate trends. In each panel, we show the log of the production rate of a particular molecule plotted against the log of the $\mathrm{CN}$ production rate. A slope equal 1 line is included on the plot to guide the eye (these lines are not fits). We see that the production rates of all of the molecules correlate well with the $\mathrm{CN}$ production rates. Except that JFCs are generally lower gas producers, we see no obvious trends with dynamical type.

\section{Trends in the data}

Figure 8 shows the observed column densities for all comets on each night they were observed, with $\mathrm{OH}, \mathrm{NH}, \mathrm{C}_{3}, \mathrm{CH}, \mathrm{C}_{2}$ and $\mathrm{NH}_{2}$ being plotted against $\mathrm{CN}$. While $\mathrm{OH}$, a daughter of the most abundant ice $\mathrm{H}_{2} \mathrm{O}$, would be the most desirable molecule to use to compare one comet against another, we chose to plot abundances versus CN because $\mathrm{CN}$ is the most commonly observed feature in our spectra. Inspection of this plot shows that the production rates of each species, including $\mathrm{OH}$, are well correlated with the production rate of $\mathrm{CN}$. Thus, comparing to $\mathrm{CN}$ is a reasonable approach. In this figure, we denote the different dynamical types of comets by different symbols. We see no obvious trend with dynamical type except that JFCs tend to produce less of all species. HTCs and LPCs seem to produce comparable amounts of gas.

Eighteen of the comets were observed on only one night each. An additional 20 comets were only observed on two nights each. The remaining 72 comets were each observed on at least three different nights or position angles, with comet 9P/Tempel 1 having 45 observations. Clearly then, some comets are dominating the trends in Figure 8. We can mitigate this effect by combining all the data for an individual comet.

In order to combine the data, we first took ratios of the production rates of each species with respect to $\mathrm{CN}$, for each date when both molecules were detected. This had the effect of removing any weather-related differences between comets, as clouds would affect all species equally. In addition, unless a comet is inhomogeneous, we expect that all the species should be produced in the same ratio at different heliocentric distances. Figure 9 shows a comparison of the change in production rates as a function of heliocentric distance versus the change in production rate ratios as a function of heliocentric distance for comet $1 \mathrm{P} /$ Halley. The production rates show a dramatic increase when the comet is at smaller heliocentric distances. In contrast, the production rate ratios are constant with heliocentric distance. Fink (1994) also found a constant mixing ratio with heliocentric distance for comet Halley.

Figure 10 shows the observed production rate ratios as a function of log heliocentric distance (using the log for the heliocentric distance just spreads out the data along the $\mathrm{x}$ axis since the vast majority of data were obtained between 1 and $2.5 \mathrm{AU}$ ). With the possible exception of $\mathrm{C}_{3}$, there are not any obvious trends with heliocentric distance, as would be expected if the species are outgassing together. We also see no obvious trends with dynamical type. The dashed lines in 


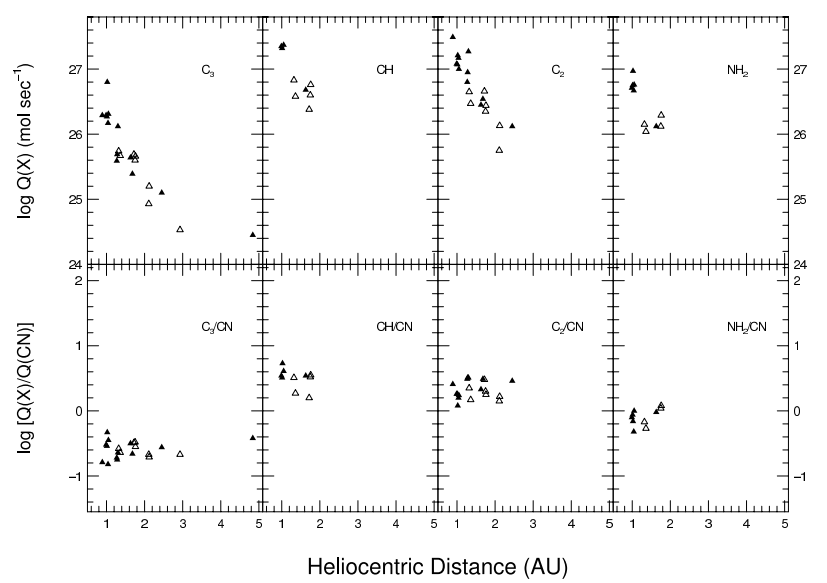

Figure 9: This figure shows how the gas production rates change as a function of heliocentric distance (upper panels) for comet Halley. We see each species showing increased production at smaller heliocentric distances. Closed symbols denote photometric nights while open symbols mean it was not photometric. In the lower panels we plot the ratio of the production rate of each species to that of $\mathrm{CN}$, with the $\mathrm{y}$ axis having the same overall range (3.8 dex) as in the upper panels. Notice that the production rate ratios are constant with heliocentric distance.

the $\mathrm{C}_{2}$ and $\mathrm{C}_{3}$ panels denote our delimiters for depleted comets, discussed below.

The $\mathrm{C}_{3}$ ratios for $\log$ heliocentric distance $>0.5$ appear to be systematically higher than the rest. There are 8 data points at these large distances but the data represent only 5 comets (there are three Hale-Bopp points). One of those is comet $1 \mathrm{P} /$ Halley. Inspection of Figure 9 suggests this point is consistent with other Halley observations at smaller heliocentric distances. Comet $\mathrm{C} / 1983$ O1 (Cernis) has the highest $\mathrm{C}_{3}$ ratio at this distance. This is the only $\mathrm{C}_{3}$ value measured for Cernis so we do not know if the comet is different or the value is high because of the large heliocentric distance. Comet C/1993 A1 (Mueller) has the next highest value but is also on the high side at $2 \mathrm{AU}$ ( $\log$ distance $=$

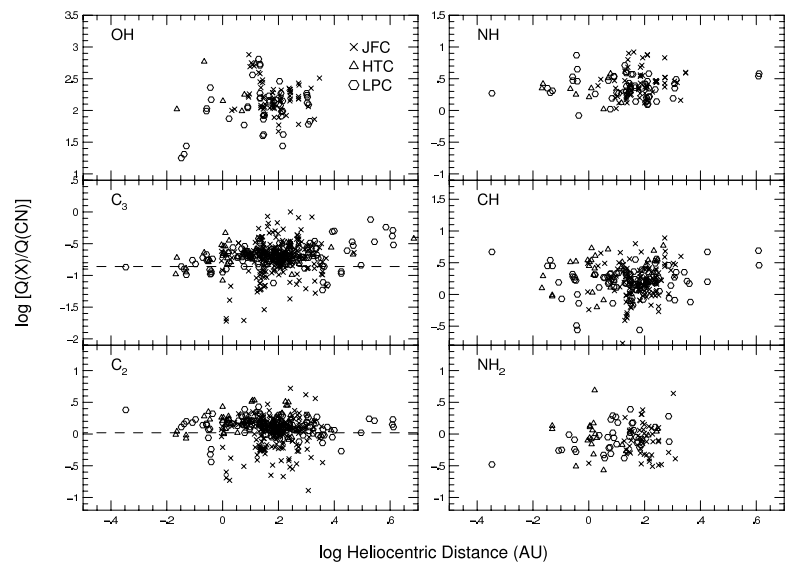

Figure 10: The production rate ratios are plotted as a function of log heliocentric distance for all instances where we observed, ratioed to $\mathrm{CN}$ for the same night. Each point represents one comet on one particular night. The different dynamical types are denoted with different symbols with the symbols defined in the $\mathrm{OH}$ panel. There are no obvious trends with heliocentric distance. The dashed lines for $\mathrm{C}_{2}$ and $\mathrm{C}_{3}$ represent the dividing line between carbon-chain typical and depleted comets (see text).

0.31). Comet $\mathrm{C} / 1996 \mathrm{P} 1$ (Wilson) has a high value of $\mathrm{C}_{3}$ at $3.5 \mathrm{AU}$ but normal-to-low values at 1.34 and $3.13 \mathrm{AU}$, suggesting the high value at $3.5 \mathrm{AU}$ may be in error. Three of the points belong to C/1995 O1 (Hale-Bopp). We only observed this comet at large heliocentric distances as part of this project so we do not know if the comet is different or there is a heliocentric distance effect.

The constancy of production rate ratios with heliocentric distance means that we can compute average values for the ratios that are representative of each comet at all observed heliocentric distances. The averages make the data for a comet with 3 observations as meaningful as a comet with 40 observations. In addition, since all species were observed simultaneously, the effects of clouds are canceled. Similarly, we have com- 


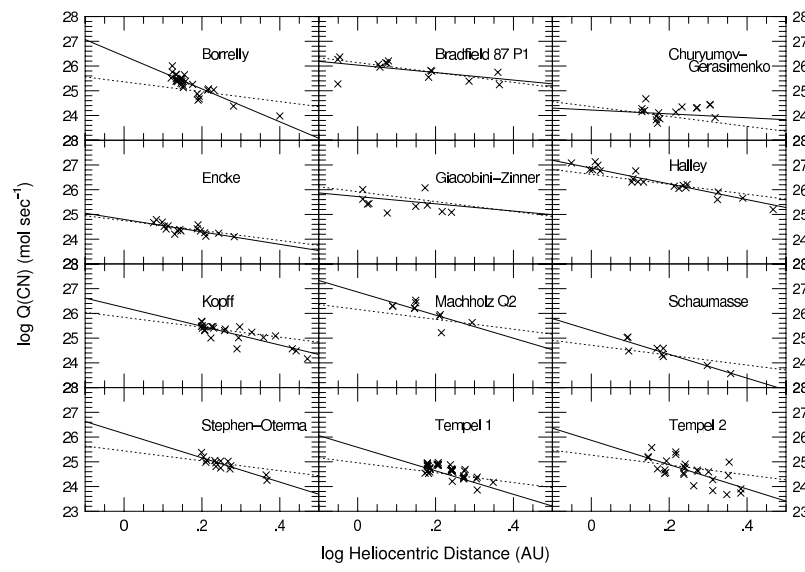

Figure 11: Twelve comets were observed at a large enough range of heliocentric distances that we could investigate how the CN production rates scale with heliocentric distance. We did not attempt to differentiate between photometric and non-photometric conditions, nor did we separate apparitions. These panels show the data along with the line that best fits the data (solid line). Also shown is a $1 / \mathrm{R}_{h}^{2}$ line (dotted line). The derived average $\mathrm{CN}$ values for $1^{\circ}$ AU using the fit and the $1 / R_{h}^{2}$ law are shown in Table 5

puted an average $\mathrm{CN}$ production for each comet by computing the production the comet would have at $1 \mathrm{AU}$ assuming a $1 / \mathrm{R}_{h}^{2}$ dependence of the production.

For the discussion that follows, it is not critical if the $\mathrm{CN}$ varies differently than we have assumed because any deviation from this law will result in more scatter in the $1 \mathrm{AU}$ values, and therefore larger error bars. The exact values are not critical to our trends. For most of our comets we did not have a large enough range of heliocentric distance to investigate the correct scaling with heliocentric distance; for twelve comets, we do cover large heliocentric distance ranges and these are shown in Figure 11. We fit a line to the data but did not in any way try to correct for weather or for changes from one apparition to the next. The solid line shows the fit while the dotted line shows a $1 / R_{h}^{2}$ line. For some comets, there is a big difference between the fit and the $1 / \mathrm{R}_{h}^{2}$ line. Table 5 lists the derived slopes and the averages from using a $1 / \mathrm{R}_{h}^{2}$ scaling versus the fitted scaling. Not surprisingly, we get different $1 \mathrm{AU} \mathrm{CN}$ values depending on what we assume. However, as we only use the $1 \mathrm{AU} \mathrm{CN}$ for Figure 12 (described below) and that value does not affect our conclusions, the exact scaling used is irrelevant, as it only moves points left or right and we care most about vertical changes. None of the other species were scaled to $1 \mathrm{AU}$; all ratios were taken without scaling species to $1 \mathrm{AU}$.

These average values are given in Table 6 . 
Table 5: The Heliocentric Dependence of the CN Production Rates

\begin{tabular}{|c|c|c|c|c|c|c|c|}
\hline Comet & \multicolumn{2}{|c|}{$\begin{array}{l}\text { Heliocentric } \\
\text { Distance }\end{array}$} & $\begin{array}{l}\text { tric } \\
\text { ce } \\
\mathrm{AU})\end{array}$ & Npts & $\begin{array}{l}\text { scaling } \\
\text { slope }\end{array}$ & $\begin{array}{c}\text { Avg. } \mathrm{Q}(\mathrm{CN}) \\
\text { at } 1 \mathrm{AU} \\
\text { (fitted) }\end{array}$ & $\begin{array}{c}\text { Avg. } \mathrm{Q}(\mathrm{CN}) \\
\text { at } 1 \mathrm{AU} \\
\left(1 / \mathrm{R}_{b}^{2}\right)\end{array}$ \\
\hline Borrelly & 1.32 & - & 2.51 & 31 & -6.6 & $4.89 \times 10^{25} \pm 3.43 \times 10^{25}$ & $2.53 \times 10^{26} \pm 1.21 \times 10^{26}$ \\
\hline Bradfield (87P1) & 0.89 & - & 2.31 & 14 & -1.5 & $1.49 \times 10^{26} \pm 9.05 \times 10^{24}$ & $1.30 \times 10^{26} \pm 2.32 \times 10^{24}$ \\
\hline CG & 1.35 & - & 2.08 & 16 & -0.8 & $4.84 \times 10^{24} \pm 3.38 \times 10^{24}$ & $2.57 \times 10^{24} \pm 1.58 \times 10^{24}$ \\
\hline Encke & 1.25 & - & 1.92 & 17 & -2.5 & $5.50 \times 10^{24} \pm 1.78 \times 10^{24}$ & $6.63 \times 10^{24} \pm 2.05 \times 10^{24}$ \\
\hline GZ & 1.03 & - & 1.74 & 10 & -1.5 & $6.63 \times 10^{25} \pm 7.41 \times 10^{25}$ & $5.71 \times 10^{25} \pm 6.07 \times 10^{25}$ \\
\hline Halley & 0.89 & - & 4.84 & 22 & -3.2 & $4.67 \times 10^{26} \pm 3.28 \times 10^{26}$ & $7.36 \times 10^{26} \pm 2.84 \times 10^{26}$ \\
\hline Kopff & 1.58 & - & 2.77 & 24 & -3.8 & $6.39 \times 10^{25} \pm 2.95 \times 10^{25}$ & $1.92 \times 10^{26} \pm 8.52 \times 10^{25}$ \\
\hline Machholz Q2 & 1.27 & - & 1.97 & 10 & -4.7 & $3.09 \times 10^{26} \pm 1.80 \times 10^{26}$ & $8.39 \times 10^{26} \pm 4.28 \times 10^{26}$ \\
\hline Schaumasse & 1.24 & - & 2.28 & 9 & -4.8 & $7.71 \times 10^{24} \pm 5.47 \times 10^{24}$ & $2.18 \times 10^{25} \pm 7.82 \times 10^{24}$ \\
\hline Stephan-Oterma & 1.58 & - & 2.33 & 14 & -4.9 & $2.79 \times 10^{25} \pm 1.27 \times 10^{25}$ & $1.45 \times 10^{26} \pm 4.32 \times 10^{25}$ \\
\hline Tempel 1 & 1.49 & - & 2.39 & 43 & -4.0 & $1.36 \times 10^{25} \pm 5.06 \times 10^{24}$ & $3.88 \times 10^{25} \pm 1.31 \times 10^{25}$ \\
\hline Tempel 2 & 1.40 & - & 2.42 & 26 & -4.9 & $2.08 \times 10^{25} \pm 2.01 \times 10^{25}$ & $1.07 \times 10^{26} \pm 1.11 \times 10^{26}$ \\
\hline
\end{tabular}


Table 6: Average Production Rate Ratios

\begin{tabular}{|c|c|c|c|c|c|c|c|c|c|c|c|c|c|c|}
\hline \multirow[t]{2}{*}{ Comet } & \multicolumn{2}{|l|}{$\log \mathrm{Q}(\mathrm{CN})$} & \multicolumn{12}{|c|}{ Average log production rate ratios } \\
\hline & & $\mathrm{N}^{a}$ & $\mathrm{OH} / \mathrm{CN}$ & $\mathrm{N}^{a}$ & & $\mathrm{~N}^{a}$ & & $\mathrm{~N}^{a}$ & $\mathrm{CH} / \mathrm{CN}$ & $\mathrm{N}^{a}$ & $\begin{array}{l}\text { Average } \\
\mathrm{C}_{2} / \mathrm{CN} \\
\end{array}$ & $\mathrm{N}^{a}$ & $\mathrm{NH}_{2} / \mathrm{CN}$ & $\mathrm{N}^{a}$ \\
\hline 49P/Arend-Rigaux & $24.56 \pm 0.47$ & 7 & & & $0.74 \pm 0.085$ & 2 & $-0.46 \pm 0.159$ & 6 & & & $0.08 \pm 0.084$ & 5 & & \\
\hline C/1982 M1 (Austin) & $25.45 \pm 0.39$ & 4 & & & & & $-0.63 \pm 0.175$ & 4 & $0.08 \pm 0.190$ & 2 & $0.19 \pm 0.029$ & 3 & $-0.25 \pm 0.007$ & 72 \\
\hline C/1984 N1 (Austin) & $25.45 \pm 0.80$ & 4 & & & & & $-0.65 \pm 0.104$ & 3 & $0.23 \pm 0.100$ & 3 & $0.18 \pm 0.083$ & 3 & -0.02 & 1 \\
\hline C/1989 X1 (Austin) & $25.64 \pm 0.17$ & 3 & 2.56 & 1 & $0.25 \pm 0.048$ & 3 & $-0.70 \pm 0.020$ & 3 & $0.31 \pm 0.048$ & 3 & $0.22 \pm 0.045$ & 3 & & \\
\hline 19P/Borrelly & $25.69 \pm 0.47$ & 31 & $2.19 \pm 0.318$ & 10 & $0.42 \pm 0.297$ & 14 & $-0.95 \pm 0.462$ & 27 & $0.01 \pm 0.314$ & 22 & $-0.19 \pm 0.096$ & 29 & $0.01 \pm 0.362$ & 11 \\
\hline 140P/Bowell-Skiff & $25.19 \pm 0.98$ & 32 & & & & & -1.09 & 1 & 0.60 & 1 & 0.19 & 1 & & \\
\hline C/1979 Y1 (Bradfield) & 25.93 & 1 & & & & & -0.87 & 1 & 0.18 & 1 & -0.05 & 1 & & \\
\hline C/1980 Y1 (Bradfield) & 26.49 & 1 & & & 0.27 & 1 & -0.87 & 1 & 0.67 & 1 & 0.37 & 1 & -0.48 & 1 \\
\hline C/1987 P1 (Bradfield) & $26.17 \pm 0.38$ & 14 & & & & & $-0.74 \pm 0.115$ & 14 & $0.26 \pm 0.124$ & 14 & $0.09 \pm 0.069$ & 14 & $-0.15 \pm 0.273$ & 312 \\
\hline $16 \mathrm{P} /$ Brooks 2 & $24.37 \pm 0.26$ & 11 & & & & & $<-0.09$ & & & & $<0.61$ & & & \\
\hline 23P/Brorsen-Metcalf & $25.67 \pm 0.28$ & 3 & $2.76 \pm 0.021$ & 2 & $0.25 \pm 0.110$ & 3 & $-0.66 \pm 0.071$ & 3 & $0.49 \pm 0.048$ & 3 & $0.24 \pm 0.031$ & 3 & -0.51 & 1 \\
\hline & $26.65 \pm 0.17$ & 2 & & & & & -0.12 & 1 & & & 0.24 & 1 & & \\
\hline $\begin{array}{l}\text { C/1990 E1 (CKN) } \\
\text { C/1980 O1 (CP) }\end{array}$ & $25.32 \pm 0.24$ & $\begin{array}{l}2 \\
1\end{array}$ & & & 0.41 & 1 & $-0.58 \pm 0.144$ & 2 & $0.06 \pm 0.174$ & 2 & $0.21 \pm 0.007$ & 2 & & \\
\hline $67 \mathrm{P} / \mathrm{CG}$ & $\begin{array}{c}24.63 \\
24.68 \pm 0.31\end{array}$ & 16 & & & & & $\begin{array}{c}<-0.16 \\
-0.58+0.203\end{array}$ & 5 & & & $\begin{array}{c}<0.81 \\
0.13 \pm 0.260\end{array}$ & 5 & & \\
\hline $71 \mathrm{P} /$ Clark & $24.97 \pm 0.38$ & 6 & & & & & $-0.71 \pm 0.129$ & 2 & & & $-0.05 \pm 0.100$ & 2 & & \\
\hline $32 \mathrm{P} /$ Comas Sola & $25.18 \pm 0.29$ & 2 & & & & & -0.88 & 1 & & & -0.89 & 1 & & \\
\hline 27P/Crommelin & $25.23 \pm 0.65$ & 58 & & & & & $-0.80 \pm 0.153$ & 7 & $0.14 \pm 0.268$ & 6 & $0.18 \pm 0.131$ & 8 & $0.26 \pm 0.509$ & 6 \\
\hline 6P/d'Arrest & $25.24 \pm 0.49$ & 16 & $2.41 \pm 0.237$ & 3 & $0.45 \pm 0.175$ & 4 & $-0.53 \pm 0.178$ & 16 & $0.33 \pm 0.405$ & 11 & $0.21 \pm 0.074$ & 15 & $-0.17 \pm 0.267$ & 74 \\
\hline $\begin{array}{l}\text { 122P/de Vico } \\
79 \mathrm{P} / \mathrm{dTH}\end{array}$ & $\begin{array}{l}26.60 \pm 0.08 \\
23.60\end{array}$ & $\begin{array}{l}3 \\
1 \\
1\end{array}$ & 2.02 & 1 & $0.39 \pm 0.050$ & 2 & $\begin{array}{l}-0.83 \pm 0.190 \\
<-0.24\end{array}$ & 2 & $0.21 \pm 0.137$ & 2 & $\begin{array}{c}0.06 \pm 0.035 \\
<0.58\end{array}$ & 2 & & \\
\hline 2P/Encke & $24.74 \pm 0.23$ & 17 & $2.56 \pm 0.225$ & 8 & $0.24 \pm 0.121$ & 8 & $-0.62 \pm 0.087$ & 17 & $0.45 \pm 0.169$ & 12 & $0.17 \pm 0.085$ & 17 & & \\
\hline $4 \mathrm{P} /$ Faye & $24.43 \pm 0.16$ & 32 & & & & & $<-0.52$ & & & & $<0.82$ & & & \\
\hline $37 \mathrm{P} /$ Forbes & $24.96 \pm 0.17$ & 2 & & & & & $<-0.75$ & & & & $<0.34$ & & & \\
\hline $78 \mathrm{P} /$ Gehrels 2 & $24.50 \pm 0.22$ & 22 & & & & & $<-0.24$ & & & & $<0.93$ & & & \\
\hline $21 \mathrm{P} / \mathrm{GZ}$ & $25.82 \pm 0.91$ & 10 & & & & & $-1.47 \pm 0.249$ & 7 & & & $-0.57 \pm 0.122$ & 9 & & \\
\hline $84 \mathrm{P} /$ Giclas & 24.83 & 1 & & & & & $<-0.71$ & & & & $<0.07$ & & & \\
\hline $26 \mathrm{P} / \mathrm{GS}$ & $24.49 \pm 0.47$ & 7 & & & & & $-0.65 \pm 0.181$ & 6 & $0.29 \pm 0.354$ & 4 & $0.01 \pm 0.227$ & 6 & -0.46 & 1 \\
\hline 促 & $25.53 \pm 0.38$ & 311 & & & & & & & & & & & & \\
\hline C/1995 O1 (Hale-Bopp) & $27.84 \pm 0.12$ & 24 & & & $0.56 \pm 0.028$ & 2 & $-0.39 \pm 0.114$ & 3 & $0.59=$ & 2 & 0.086 & 3 & & \\
\hline 1P/Halley & $26.67 \pm 0.51$ & 25 & & & & & $-0.57 \pm 0.129$ & 23 & $0.49 \pm 0.165$ & 12 & $0.34 \pm 0.160$ & 21 & $-0.08 \pm 0.128$ & 810 \\
\hline 51P/Harrington & 24.77 & 1 & & & & & -0.74 & 1 & & & 0.17 & 1 & & \\
\hline 52P/Harrington-Abell & $24.51 \pm 0.25$ & 53 & & & & & $<-0.43$ & & & & $<0.62$ & & & \\
\hline $\mathrm{C} / 1995$ Q2 (HD) & $24.42 \pm 0.00$ & 1 & & & & & $<-0.39$ & & & & $<0.24$ & & & \\
\hline 1985 R1 (Hartley-Good) & $25.40 \pm 0.24$ & 44 & & & & & $-0.68 \pm 0.033$ & 4 & & & $0.11 \pm 0.018$ & 4 & $0.20 \pm 0.125$ & 4 \\
\hline 161P/Hartley-IRAS & $25.37 \pm 0.48$ & 32 & & & & & -0.70 & 1 & & & 0.11 & 1 & 0.26 & 1 \\
\hline $111 \mathrm{P} / \mathrm{HRC}$ & 25.66 & 1 & & & & & $<-0.21$ & & & & $<0.70$ & & & \\
\hline $45 \mathrm{P} / \mathrm{HMP}$ & 24.31 & 1 & 2.21 & 1 & & & -0.93 & 1 & & & 0.19 & 1 & & \\
\hline 88P/Howell & $25.00 \pm 0.31$ & 13 & & & & & $-0.25 \pm 0.365$ & 2 & & & 0.24 & 1 & & \\
\hline C/1995 Y1 (Hyakutake) & 25.41 & 1 & 1.87 & 1 & 0.26 & 1 & -0.80 & 1 & 0.31 & 1 & 0.16 & 1 & & \\
\hline C/1996 B2 (Hyakutake) & $26.73 \pm 0.68$ & 3 & $2.13 \pm 0.035$ & 52 & $0.49 \pm 0.087$ & 3 & $-0.60 \pm 0.030$ & 3 & $0.36 \pm 0.035$ & 2 & $0.29 \pm 0.005$ & 3 & & \\
\hline $153 \mathrm{P} / \mathrm{Ike}$ & $26.48 \pm 0.14$ & 13 & $1.34 \pm 0.102$ & 3 & $0.31 \pm 0.035$ & 3 & $-0.91 \pm 0.065$ & 3 & $0.48 \pm 0.054$ & 3 & $0.17 \pm 0.030$ & 3 & & \\
\hline $\mathrm{C} / 1983 \mathrm{H}$ & $25.26 \pm 0.14$ & 3 & & & & & $-0.66 \pm 0.025$ & 3 & & & $0.04 \pm 0.052$ & 3 & $0.12 \pm 0.014$ & 2 \\
\hline $126 \mathrm{P} / \mathrm{IR}$ & $24.99 \pm 0.48$ & 4 & & & & & $<-0.57$ & & & & $<0.36$ & & & \\
\hline C/1983 O2 (IRAS) & 24.85 & 1 & & & & & $<-0.29$ & & & & $<0.88$ & & & \\
\hline 58P/Jackson-Neujmin & $23.90 \pm 0.27$ & 5 & 2 & 1 & \pm 0 & 2 & $-0.24 \pm 0.147$ & 5 & & & $0.26 \pm 0.193$ & 4 & & \\
\hline 59P/Kearns-Kwee & $25.36 \pm 0.23$ & 10 & $2.51 \pm 0.272$ & 1 & $0.60 \pm 0.007$ & 2 & $-0.77 \pm 0.241$ & 8 & & & $0.14 \pm 0.230$ & 8 & & \\
\hline 68P/Klemola & $24.87 \pm 0.55$ & 54 & & & & & $-0.81 \pm 0.290$ & 3 & & & $0.47 \pm 0.164$ & 3 & & \\
\hline $75 \mathrm{P} /$ Kohoutek & $25.30 \pm 0.16$ & 4 & & & & & $-0.67 \pm 0.127$ & 4 & $0.25 \pm 0.260$ & 4 & $-0.10 \pm 0.136$ & 4 & $-0.30 \pm 0.172$ & 24 \\
\hline
\end{tabular}




\begin{tabular}{|c|c|c|c|c|c|c|c|c|c|c|c|c|c|c|}
\hline \multirow[t]{2}{*}{ Comet } & \multicolumn{2}{|l|}{$\log \mathrm{Q}(\mathrm{CN})$} & \multicolumn{12}{|c|}{ Average log production rate ratios } \\
\hline & at $1 \mathrm{AU}$ & $\mathrm{N}^{a}$ & $\mathrm{OH} / \mathrm{CN}$ & $\mathrm{N}^{a}$ & $\mathrm{NH} / \mathrm{CN}$ & $\mathrm{N}^{a}$ & $\mathrm{C}_{3} / \mathrm{CN}$ & $\mathrm{N}^{a}$ & $\mathrm{CH} / \mathrm{CN}$ & $\mathrm{N}^{a}$ & $\begin{array}{l}\text { Average } \\
\mathrm{C}_{2} / \mathrm{CN}\end{array}$ & $\mathrm{N}^{a}$ & $\mathrm{NH}_{2} / \mathrm{CN}$ & $\mathrm{N}^{a}$ \\
\hline $22 \mathrm{P} / \mathrm{Kopff}$ & $25.81 \pm 0.30$ & $\sqrt{24}$ & $1.79 \pm 0.113$ & 3 & $0.27 \pm 0.124$ & $\frac{3}{3}$ & $-0.74 \pm 0.086$ & 210 & $0.19 \pm 0.164$ & 14 & & 20 & $-0.11 \pm 0.178$ & 9 \\
\hline $144 \mathrm{P} /$ Kushida & $25.27 \pm 0.16$ & 43 & $2.01 \pm 0.177$ & 4 & $0.28 \pm 0.014$ & 2 & $-0.61 \pm 0.077$ & 40 & $0.20 \pm 0.122$ & 2 & $0.13 \pm 0.038$ & 4 & & \\
\hline C/1987 A1 (Levy) & $24.74 \pm 0.39$ & 3 & & & & & $-0.61 \pm 0.018$ & 30 & $0.01 \pm 0.190$ & 2 & $0.14 \pm 0.071$ & 3 & $-0.02 \pm 0.057$ & 2 \\
\hline C/1990 K1 (Levy) & $26.36 \pm 0.20$ & 5 & $2.75 \pm 0.055$ & 3 & $0.60 \pm 0.098$ & 4 & $\begin{array}{l}-0.01 \pm 0.018 \\
-0.46 \pm 0.142\end{array}$ & 50 & $0.32 \pm 0.089$ & 4 & $0.32 \pm 0.075$ & 5 & $-0.02 \pm 0.058$ & \\
\hline C/1984 V1 (LR) & $25.21 \pm 0.24$ & 6 & & & & & $-0.69 \pm 0.087$ & 60 & $0.13 \pm 0.287$ & 5 & $0.06 \pm 0.056$ & 5 & $0.09 \pm 0.096$ & 5 \\
\hline C/1988 A1 (L & $3.32 \pm 0.22$ & 5 & & & & & $-0.66 \pm 0.056$ & 50 & $0.09 \pm 0.102$ & 5 & $0.01 \pm 0.071$ & & $-0.12 \pm 0.043$ & 5 \\
\hline C/2001 A2 (LII & 25.32 & 1 & & & & & $\begin{array}{l}-0.00 \pm 0.000 \\
-0.68\end{array}$ & 1 & $0.09 \pm 0.102$ & 0 & $\begin{array}{l}0.01 \\
0.27\end{array}$ & & & \\
\hline C/2000 WM1 (LINEAR) & $25.78 \pm 0.13$ & 5 & 2.46 & 1 & $0.47 \pm 0.220$ & 3 & $-0.89 \pm 0.136$ & 50 & $0.02 \pm 0.159$ & 2 & $0.02 \pm 0.117$ & 5 & & \\
\hline C/2006 VZ13 (LINEAR) & $25.35 \pm 0.35$ & 2 & $1.61 \pm 0.014$ & 2 & $0.37 \pm 0.093$ & 2 & $-0.67 \pm 0.028$ & 2 & 0.15 & 1 & $0.10 \pm 0.025$ & 2 & & \\
\hline 93P/Lovas 1 & $24.54 \pm 0.17$ & 2 & & & & & $-0.40 \pm 0.042$ & 2 & & & $0.13 \pm 0.375$ & 2 & & \\
\hline C/2007 E2 (Lovejoy) & 25.07 & 2 & $1.54 \pm 0.129$ & 2 & $0.29 \pm 0.093$ & 2 & $-0.78 \pm 0.035$ & 2 & & & $0.07 \pm 0.024$ & 2 & & \\
\hline C/1994 T1 (Machholz) & $25.55 \pm 0.08$ & 42 & $2.21 \pm 0.062$ & 4 & 0.19 & 1 & $-0.62 \pm 0.080$ & 3 & & & $0.21 \pm 0.042$ & 4 & & \\
\hline C/2004 Q2 (Machholz) & $26.49 \pm 0.34$ & 10 & $1.96 \pm 0.067$ & 9 & $0.33 \pm 0.174$ & 9 & $-0.74 \pm 0.062$ & 100 & $0.22 \pm 0.050$ & 10 & $0.16 \pm 0.035$ & 10 & & \\
\hline 141P/Machholz 2-A & $24.11 \pm 0.06$ & 2 & $2.01 \pm 0.014$ & 2 & $0.44 \pm 0.071$ & 2 & $-0.67 \pm 0.064$ & 20 & $0.56 \pm 0.144$ & 2 & $0.13 \pm 0.019$ & 2 & & \\
\hline $\begin{array}{c}\text { 115P/Maury } \\
\text { C/1993 Y1 (MR) }\end{array}$ & $\begin{array}{c}25.74 \\
25.03 \pm 0.61\end{array}$ & $\begin{array}{l}1 \\
7\end{array}$ & $2.11 \pm 0.241$ & 5 & $0.52 \pm 0.359$ & 7 & $\begin{array}{c}<-0.56 \\
-0.83 \pm 0.129\end{array}$ & $\begin{array}{lll}7 & 0\end{array}$ & $0.02 \pm 0.414$ & 6 & $\begin{array}{c}<0.27 \\
0.05 \pm 0.264\end{array}$ & 7 & & \\
\hline C/1980 V1 (Meier) & $26.22 \pm 0.47$ & 6 & & & $0.25 \pm 0.137$ & 2 & $-0.71 \pm 0.165$ & 60 & $0.29 \pm 0.371$ & 5 & $0.14 \pm 0.020$ & 5 & 0.11 & 1 \\
\hline C/1993 A1 & $26.18 \pm 0.61$ & 2 & 1.83 & 1 & 0.49 & 1 & $-0.35 \pm 0.182$ & 2 & 0.35 & 1 & 0.12 & & & \\
\hline $28 \mathrm{P} / \mathrm{N}$ & $24.62 \pm 0.25$ & 2 & & & & & -0.17 & 1 & & & 0.27 & 1 & & \\
\hline C/1987 B1 (NTT) & $25.62 \pm 0.31$ & 3 & & & & & $-0.63 \pm 0.135$ & 30 & $0.25 \pm 0.050$ & 2 & $0.23 \pm 0.176$ & 3 & $0.11 \pm 0.205$ & 2 \\
\hline $\mathrm{C} / \mathbf{1 9 8 0} \mathrm{Y2}(\mathrm{P}$ & $49 \pm 0.24$ & 4 & & & & & $\begin{array}{c}<-0.42 \\
-0.89 \pm 0.181\end{array}$ & 3 & & & $\begin{array}{r}0.2 \\
0.13 \pm\end{array}$ & $\begin{array}{l}1 \\
3\end{array}$ & & \\
\hline $80 \mathrm{P} /$ Peters-Hartley & $25.24 \pm 0.14$ & 2 & & & & & $-0.76 \pm 0.122$ & $\begin{array}{ll}2 & -1 \\
2 & -1\end{array}$ & $-0.23 \pm 0.057$ & 2 & $0.15 \pm 0.189$ & 2 & $0.01 \pm 0.144$ & 2 \\
\hline $83 \mathrm{P} /$ Russell 1 & $26.13 \pm 0.36$ & 3 & & & & & $<-0.40$ & & & & $<0.71$ & & & \\
\hline 91P/Russell 3 & 24.53 & 1 & & & & & $<-0.04$ & & & & $<0.75$ & & & \\
\hline $24 \mathrm{P} /$ Schaumasse & $24.89 \pm 0.77$ & 92 & $2.48 \pm 1.398$ & 4 & $0.35 \pm 0.136$ & 3 & $-0.76 \pm 0.159$ & 80 & $0.11 \pm 0.195$ & 3 & $0.21 \pm 0.349$ & 8 & $-0.08 \pm 0.272$ & 2 \\
\hline $106 \mathrm{P}$ & 23.99 & 1 & & & & & & & & & $<0.61$ & & & \\
\hline $31 \mathrm{P} / \mathrm{SW} 2$ & $25.61 \pm 0.16$ & 62 & $2.17 \pm 0.397$ & 2 & $0.32 \pm 0.205$ & 2 & $-0.75 \pm 0.174$ & 2 & & & $-0.20 \pm 0.156$ & 2 & & \\
\hline Demaker 1 & $26.11 \pm 0.45$ & 3 & & & & & $-0.71 \pm 0.288$ & 3 & 0.49 & 1 & $0.03 \pm 0.204$ & 3 & 0.64 & 1 \\
\hline $\mathrm{C} / 1 \mathrm{~S}$ & $26.13 \pm 0.35$ & 2 & & & & & -0.47 & 1 & & & $<0.51$ & & & \\
\hline $\mathrm{C} / 1984 \mathrm{U} 2$ & $24.58 \pm 0.21$ & 2 & & & & & $<-0.84$ & & & & $<-0.04$ & & & \\
\hline $\mathrm{C} / 1 \mathrm{~s}$ & 26.85 & 1 & & & & & $<0.21$ & & & & $<1.00$ & & & \\
\hline C/1989 A6 (Shoe & 25.73 & 1 & & & & & -0.31 & 1 & & & -0.05 & 1 & & \\
\hline $\mathrm{C} / 1988 \mathrm{~J} 1 \mathrm{SH}$ & 25.25 & 1 & & & & & $\begin{array}{l}-0.01 \\
-0.57\end{array}$ & 1 & & & $\begin{array}{l}-0.00 \\
-0.12\end{array}$ & 1 & & \\
\hline -Levy 1 & 24.1 & 1 & & & & & $<-0.72$ & & & & $<0.13$ & & & \\
\hline $\mathrm{C} / \mathbf{2}$ & $25.49 \pm 0.04$ & 32 & $2.20 \pm 0.097$ & 3 & $0.19 \pm 0.032$ & 3 & $-0.76 \pm 0.050$ & 30 & 0.2 & 3 & $0.20 \pm 0.015$ & & & \\
\hline C/ & $26.15 \pm$ & 3 & & & & & & 30 & & & 32 & & & \\
\hline $38 \mathrm{P} /$ & $25.44 \pm 0.30$ & 14 & & & & & 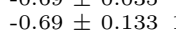 & 120 & $0.56 \pm 0.231$ & 5 & 74 & 11 & & \\
\hline $\mathrm{C} / 1$ & $24.29 \pm 0.01$ & 2 & & & & & $-0.92 \pm 0.035$ & & & & $0.14 \pm 0.057$ & 2 & & \\
\hline $64 \mathrm{P} / \mathrm{s}$ & $24.94 \pm 0.50$ & 10 & & & & & $-0.68 \pm 0.057$ & 100 & $0.13 \pm$ & 2 & $0.14 \pm 0.080$ & $8-$ & $-0.07 \pm 0.158$ & 3 \\
\hline 109 & 26.86 & 1 & 2.1 & 1 & 0.2 & 1 & -0.66 & 1 & 0.4 & 1 & 0.23 & 1 & & \\
\hline C/1996 B1 (Szczepanski) & $25.91 \pm 0.24$ & 32 & $2.18 \pm 0.053$ & 3 & $0.31 \pm 0.127$ & 3 & $-0.75 \pm 0.039$ & 30 & $0.35 \pm 0.243$ & 3 & $0.07 \pm 0.015$ & 3 & & \\
\hline $\begin{array}{l}\text { 98P/Takamizawa } \\
\text { C/1994 J2 (Takamizawa) }\end{array}$ & $\begin{array}{c}24.54 \\
25.80 \pm 0.14\end{array}$ & $\begin{array}{l}1 \\
2\end{array}$ & & 2 & & 2 & $\begin{array}{c}<-0.14 \\
-0.69 \pm 0.035\end{array}$ & 20 & & 2 & $0.08+0.085$ & 2 & & \\
\hline C/1994 G1-A (TL) & $25.60 \pm 0.24$ & 3 & $2.22 \pm 0.045$ & 3 & $0.33 \pm 0.049$ & 3 & $-0.69 \pm 0.023$ & 30 & $0.29 \pm 0.099$ & 3 & $0.16 \pm 0.012$ & 3 & & \\
\hline $69 \mathrm{P}$ & $24.67 \pm 0.34$ & 5 & & & & & $<-0.35$ & & & & $<0.60$ & & & \\
\hline & $25.13 \pm 0.21$ & 432 & $2.21 \pm$ & & & & & 40 & & 31 & & & & \\
\hline & & 26 & & & & & & 18 & & & 2 & & & 8 \\
\hline & 25.1 & & & & & & & & $0.51 \pm 0.540$ & & & & & \\
\hline
\end{tabular}


In Table 6 we include the comet name (sometimes abbreviated to save space; the abbreviations are at the bottom of the table), the average $\mathrm{CN}$ production rate at $1 \mathrm{AU}$ and the average $\log [\mathrm{Q}(\mathrm{X}) / \mathrm{Q}(\mathrm{CN})]$ where $\mathrm{X}$ represents the various observed species. We also include the standard deviations of the averages (when more than one data point was present). The number of data points going into each average is listed. When we did not observe a species (either because it was not in our bandpass or we could not measure it) the value in the table is either the upper limit (for $\mathrm{C}_{2}$ or $\mathrm{C}_{3}$ as described above) or is left blank.

As noted above, a number of comets were observed on only one or two nights. While these observations may have been of extremely high quality (comet 122/de Vico is such an example), many of the single or double night observations were also of faint and less well observed comets. Thus, in a manner similar to AH95, we created a restricted data set for exploration of the data. For our purposes, we define a comet as being a member of the restricted data set if it was observed on at least three different nights (or night/position angle combinations). There are 72 comets that meet this criterion. However, of these 72 comets, 13 had only upper limits for $\mathrm{C}_{2}$ and/or $\mathrm{C}_{3}$. These upper limits are not constraining and so we dropped these 13 comets from our definition of the restricted data set. Thus, our restricted data set consists of the remaining 59 comets. In Table 6 these comets are listed with their names left-justified and bold-faced. The 18 single-observation and 20 double-observation comets are indented and not bold-faced, as are the 13 comets with more than 2 observations but only upper limits for $\mathrm{C}_{3}$ and/or $\mathrm{C}_{2}$. This definition of restricted data set is somewhat arbitrary and does not attempt to take into account the

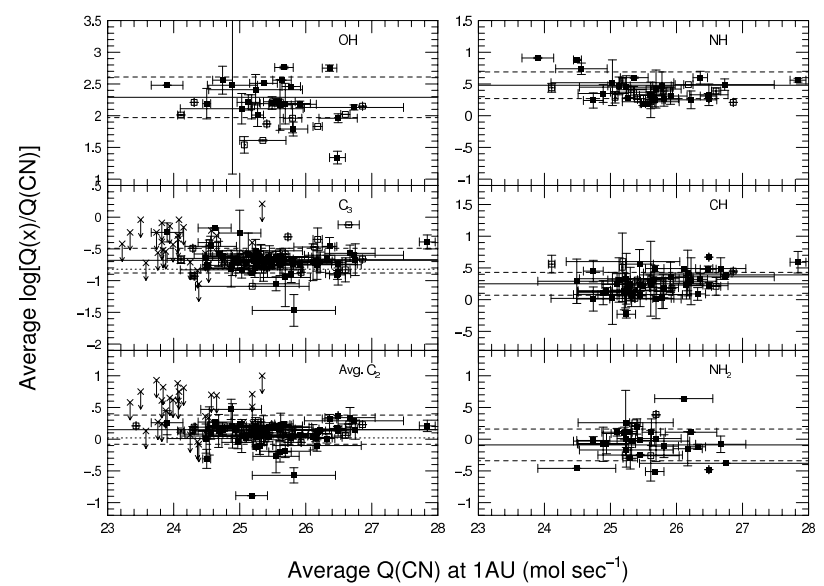

Figure 12: The average production rate ratios for each comet and each molecule are shown plotted against the average $\mathrm{Q}(\mathrm{CN})$ at $1 \mathrm{AU}$. In each panel, the filled symbols are comets in the restricted data set; the open symbols are the data not in the restricted data set. For $\mathrm{C}_{2}$ and $\mathrm{C}_{3}$, the upper limits are shown for comets with detections of $\mathrm{CN}$ but no $\mathrm{C}_{2}$ or $\mathrm{C}_{3}$ detection. The solid horizontal line is the weighted mean of the production rate ratios. The dashed lines represent the standard deviations from the means. For $\mathrm{C}_{2}$ and $\mathrm{C}_{3}$, the dotted lines mark the boundary between typical and depleted comets. Details of this figure are discussed in the text.

quality of the spectra. However, it is an unbiased approach to choosing the restricted set.

Figure 12 shows the production rate ratios of all comets as a function of the $1 \mathrm{AU} \mathrm{CN}$ production rate. While it would have been preferable to plot against $\mathrm{CN} / \mathrm{OH}$, we did not observe $\mathrm{OH}$ on enough dates to use the $\mathrm{CN} / \mathrm{OH}$ data in the comparison. AH95 showed that $\mathrm{CN}$ correlates well with $\mathrm{OH}$ (their figure 9) for all types of comets. The data from the restricted data set are shown as filled symbols, while the data that are not in the restricted data set are shown with open symbols The upper limits for $\mathrm{C}_{3}$ and $\mathrm{C}_{2}$ discussed above are shown on the appropriate panels. As indicated above, almost none of these limits is very constraining. When we did not detect a 
species, it did not necessarily mean that there was none of that molecule, just that the noise was high. Included in Figure 12 are the weighted means and standard deviations for the detections in the restricted data set for the quantities on the y axes. These means and standard deviations were computed using all 59 of the comets in the restricted data set, without consideration of extreme outliers.

The scatter in the $\mathrm{Q}(\mathrm{OH}) / \mathrm{Q}(\mathrm{CN})$ is quite high (confirmed by the standard deviation being the largest we have) and it is difficult to reach any conclusions based on these data. This is another reason why we chose not to compare all the comets with ratios to $\mathrm{OH}$ production. For the comets in the restricted data set, the outliers are 23P/Brorsen-Metcalf and C/1990 K1 (Levy) on the high side and 22P/Kopff and 153P/IkeyaZhang on the low side. It is Ikeya-Zhang that is the extremely low point.

$\mathrm{Q}(\mathrm{NH}) / \mathrm{Q}(\mathrm{CN})$ is restricted to a very narrow range. The three high data points are 49P/Arend-Rigaux, 58P/Jackson-Neujmin and 76P/West-Kohoutek-Ikemura. These are of sufficiently good quality that they actually move the weighted mean upward over what the eye would choose. There are a number of comets below the bottom standard deviation line. C/1994 T1 (Machholz) and C/2002 E2 (Snyder-Murakami) are the most deviant. Five other comets are within 0.05 of the lower standard deviation.

$\mathrm{Q}\left(\mathrm{NH}_{2}\right) / \mathrm{Q}(\mathrm{CN})$ shows more scatter than $\mathrm{Q}(\mathrm{NH}) / \mathrm{Q}(\mathrm{CN})$, though one would assume that $\mathrm{NH}_{2}$ dissociates to NH. Generally, we only observed the $\mathrm{NH}_{2}$ with the IDS (with a few exceptions) while we observed the $\mathrm{NH}$ with the LCS. Rarely are the two species observed in the same comet due to the wavelength range covered in the spectra. Inspection of Table 6 shows that the error bars are generally quite a bit higher for the detections of $\mathrm{NH}_{2}$. The highest $\mathrm{Q}\left(\mathrm{NH}_{2}\right) / \mathrm{Q}(\mathrm{CN})$ is for comet $102 \mathrm{P} /$ Shoemaker 1 , which had only one $\mathrm{NH}_{2}$ detection when the comet was at $2.01 \mathrm{AU}$ and no $\mathrm{NH}$ measurement. 27P/Crommelin and 1985 R1 (Hartley-Good) are slightly elevated in $\mathrm{NH}_{2}$. The lowest outliers are 23P/Brorsen-Metcalf, C/1980 Y1 (Bradfield), 26P/Grigg-Skjellerup and C/1986 P1 (Wilson). These comets had only one $\mathrm{NH}_{2}$ detection each. $\mathrm{NH}_{2}$ is a very difficult feature to observe since it is very weak and diffuse. Comets Brorsen-Metcalf and Bradfield both also had NH observations. Both were at the lower edge or just below the NH normal band.

$\mathrm{Q}(\mathrm{CH}) / \mathrm{Q}(\mathrm{CN})$ seems to show many comets that are off the average by just about the standard deviation of the average. However, there are few extreme outliers. Most of the extreme comets, such as $\mathrm{C} / 1980 \mathrm{Y} 1$ (Bradfield), with $\log [\mathrm{Q}(\mathrm{CH}) / \mathrm{Q}(\mathrm{CN})]=0.67$, have only 1 data point. However, 2P/Encke, 1P/Halley, C/1993 Y1 (McNaught-Russell), and $38 \mathrm{P} /$ Stephan-Oterma have many data points. All of these comets are at the high end, except for McNaught-Russell. 23P/Brorsen-Metcalf has three data points and is also high. The lowest data point is comet $80 \mathrm{P} /$ Peters-Hartley, with two data points when the comet was at $1.78 \mathrm{AU}$.

If we ignore the upper limits, $\mathrm{Q}\left(\mathrm{C}_{2}\right) / \mathrm{Q}(\mathrm{CN})$ shows a very tight correlation for most comets but some comets show significant deviations. The high data point is $68 \mathrm{P} /$ Klemola. The low outliers are comets 19P/Borrelly, 32P/Comas Sola, 21P/Giacobini-Zinner, 75P/Kohoutek, C/1988 J1 (Shoemaker-Holt), 31P/Schwassmann-Wachmann 2, 76P/WestKohoutek-Ikemura, 81P/Wild 2, and C/1989 A1 (Yanaka). Most of these are well observed. The two lowest comets are Giacobini-Zinner (-0.57) 
and Comas Sola (-0.89).

$\mathrm{Q}\left(\mathrm{C}_{3}\right) / \mathrm{Q}(\mathrm{CN})$ also shows high scatter, though most of the upper limits are not very constraining. The high outliers are $49 \mathrm{P} /$ ArendRigaux, C/1995 O1 (Hale-Bopp), 88P/Howell, 58P/Jackson-Neujmin, C/1990 K1 (Levy), and 28P/Neujmin 1. Neujmin 1, Jackson-Neujmin and Howell are the three highest values. On the low side, $21 \mathrm{P} /$ Giacobini-Zinner is the extremely low point, with $\log \left[\mathrm{Q}\left(\mathrm{C}_{3}\right) / \mathrm{Q}(\mathrm{CN})\right]=-$ 1.47. The other low points (values less than -0.9) are 19P/Borrelly, 153P/Ikeya-Zhang, and 81P/Wild 2.

Categorizing individual comets was not the point of our program. We sought to understand whether comets could have been formed with more than one compositional type. This is not the first study of its kind. In addition to the papers by AH95, F09 and LS11 listed in the introduction, smaller studies were carried out by A'Hearn and Millis (1980), Newburn and Spinrad (1984), Fink and Hicks (1996) and by our group (Cochran 1987; Cochran et al. 1987, 1989, 1992; Cochran and Barker 1987). Thus, it has already been discovered that, while the spectra of most comets are very similar, some comets show much weaker $\mathrm{C}_{2}$ and $\mathrm{C}_{3}$ relative to other species. AH95 used a database of 85 comets to quantify this trend and have defined two taxonomic classes: typical and carbon-chain depleted (shortened to depleted).

In order to study the abundance correlations of the various species, we started by forming weighted means and standard deviations of the data. These are listed in Table 7. In this table, we list the values for each species over various subsets of the data. First, we computed the mean for all 110 comets with CN observations (upper limits were not used in the means). We also list the means and standard deviations for the complete restricted data set. Inspection of Table 7 shows that the restricted data set is representative of the whole data set. The complete restricted data set's means and standard deviations are shown as solid and dashed lines, respectively, in Figure 12 .

Using the values from the complete data set, we have formed a definition for a depleted comet in our data set. A depleted comet is one for which $\log \left[Q\left(C_{3}\right) / Q(C N)\right] \leq-0.86$ and $\log \left[Q\left(C_{2}\right) / Q(C N)\right] \leq 0.02$. Note that we require both the $\mathrm{C}_{3}$ and $\mathrm{C}_{2}$ ratios to be low. These values are shown in Figure 12 as dotted lines in the $\mathrm{C}_{2}$ and $\mathrm{C}_{3}$ panels. Within the restricted data set we find 5 comets that fit this definition: 19P/Borrelly, 32P/Comas Sola, 21P/Giacobini-Zinner, C/2000 WM1 (LINEAR), and 81P/Wild 2. Borrelly, GiacobiniZinner and Wild 2 have been previously shown to be depleted by us and others. Thus, in our restricted data set we find $5 / 59$ comets belong to the carbon-chain depleted group, or $9 \%$. For the comets not in the restricted set, C/1979 Y1 (Bradfield) is also depleted. In addition, while most of the upper limits are not constraining, two of the comets with only upper limits have values that are below the defined cutoff for depleted comets and therefore must be depleted: C/1984 U2 (Shoemaker) and 43P/WolfHarrington (both were found depleted by AH95).

For these depleted comets, the depletion of $\mathrm{C}_{2}$ and $\mathrm{C}_{3}$ is not a subtle effect. Figure 13 shows spectra of $21 \mathrm{P} /$ Giacobini-Zinner and $8 \mathrm{P} /$ Tuttle, obtained with the same instrument. The viewing geometry for these two observations is almost identical, with only a very slight difference in heliocentric distance. The two spectra are scaled by the CN band. The Tuttle spectrum shows all of the usual molecules while the GiacobiniZinner spectrum is "missing" the $\mathrm{C}_{2}$ and $\mathrm{C}_{3}$. In- 
Table 7: Derived Production Rate Ratios

\begin{tabular}{ccccccc}
\hline & \multicolumn{5}{c}{ Log Production Rate Ratio with respect to CN } \\
\cline { 2 - 7 } & $\mathrm{OH}$ & $\mathrm{NH}$ & $\mathrm{C}_{3}$ & $\mathrm{CH}$ & $\begin{array}{c}\mathrm{C}_{2} \\
\text { (average) }\end{array}$ & $\mathrm{NH}_{2}$ \\
\hline All Comets & $2.09 \pm 0.35$ & $0.46 \pm 0.19$ & $-0.68 \pm 0.20$ & $0.25 \pm 0.19$ & $0.15 \pm 0.20$ & $-0.09 \pm 0.27$ \\
$\quad(110$ comets $)$ & $(33$ comets $)$ & $(38$ comets $)$ & $(84$ comets $)$ & $(41$ comets $)$ & $(84$ comets $)$ & $(29$ comets $)$ \\
All Restricted Comets & $2.29 \pm 0.32$ & $0.48 \pm 0.21$ & $-0.68 \pm 0.19$ & $0.25 \pm 0.18$ & $0.15 \pm 0.23$ & $-0.09 \pm 0.25$ \\
$\quad(59$ comets) & $(23$ comets $)$ & $(27$ comets $)$ & $(59$ comets $)$ & $(41$ comets $)$ & $(59$ comets $)$ & $(26$ comets $)$ \\
\hline Restricted, Typical & $2.29 \pm 0.33$ & $0.48 \pm 0.21$ & $-0.67 \pm 0.15$ & $0.25 \pm 0.18$ & $0.16 \pm 0.14$ & $-0.09 \pm 0.26$ \\
$\quad(54$ comets) & $(21$ comets $)$ & $(25$ comets $)$ & $(54$ comets $)$ & $(39$ comets $)$ & $(54$ comets $)$ & $(25$ comets $)$ \\
Restricted, Depleted & $2.19 \pm 0.16$ & $0.44 \pm 0.28$ & $-1.06 \pm 0.39$ & $0.02 \pm 0.26$ & $-0.24 \pm 0.60$ & 0.01 \\
$\quad(5$ comets $)$ & $(2$ comets $)$ & $(2$ comets $)$ & $(5$ comets $)$ & $(2$ comets $)$ & $(5$ comets $)$ & $(1$ comet $)$ \\
\hline
\end{tabular}

Depleted comet is defined as $\log \left[\mathrm{Q}\left(\mathrm{C}_{3}\right) / \mathrm{Q}(\mathrm{CN})\right] \leq-0.86$ and $\log \left[\mathrm{Q}\left(\mathrm{C}_{2}\right) / \mathrm{Q}(\mathrm{CN})\right] \leq 0.02$.

spection of the Giacobini-Zinner spectrum shows we detected the weak $\mathrm{CN} \Delta v=-1$ band at $\sim 4200 \AA$, so the failure to detect the $\mathrm{C}_{2}$ and $\mathrm{C}_{3}$ is not a failure of signal/noise. GiacobiniZinner really does have very little of these two

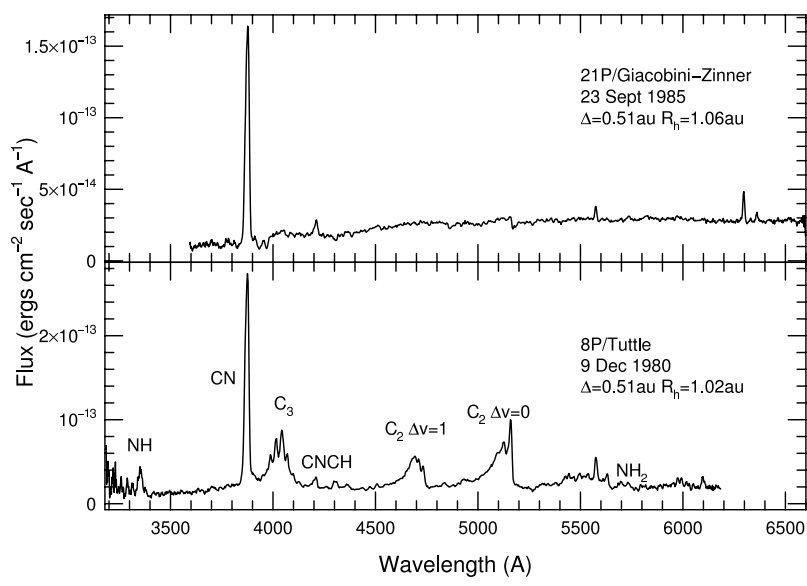

Figure 13: IDS Spectra of 21P/Giacobini-Zinner and $8 \mathrm{P} /$ Tuttle are shown when both comets were near a heliocentric distance of $1 \mathrm{AU}$ and a geocentric distance of $0.5 \mathrm{AU}$. While both comets show similar $\mathrm{CN}$ (including the weaker $\Delta v=-1$ band near $\sim 4200 \AA$ ), there is little evidence of the $\mathrm{C}_{3}$ or $\mathrm{C}_{2}$ bands in the Giacobini-Zinner spectrum. molecules!

In Table 7 we list means and standard deviations for the 54 comets in the restricted data set that are not depleted ("typical") and separately list these values for the 5 depleted comets. Comparison of these two groups shows that, while the depleted comets are significantly different in their $\mathrm{C}_{3}$ and $\mathrm{C}_{2}$ ratios, $\mathrm{OH}$ and $\mathrm{NH}$ look the same in both sets. $\mathrm{CH}$ shows a little difference between the typical and depleted comets, but the error bars are high. $\mathrm{NH}_{2}$ has too little data (only 1 comet) in the depleted group to draw any conclusion.

As noted above, other groups have looked at abundance correlations between comets. As each study has used slightly different fluorescence efficiencies and Haser scale lengths, one cannot simply compare the production rate ratios from one study with another study. However, as AH95 pointed out "...the pattern of values of the ratios will, to first order, be independent of these parameters".

Figure 14 illustrates the effects of using different scale lengths for computing the column densities. It shows the $\mathrm{CN}$ and $\mathrm{C}_{2}$ column densities 
for two different comets along with model fits using the scale lengths of AH95, F09, LS11 and the values used in this paper. These two particu-

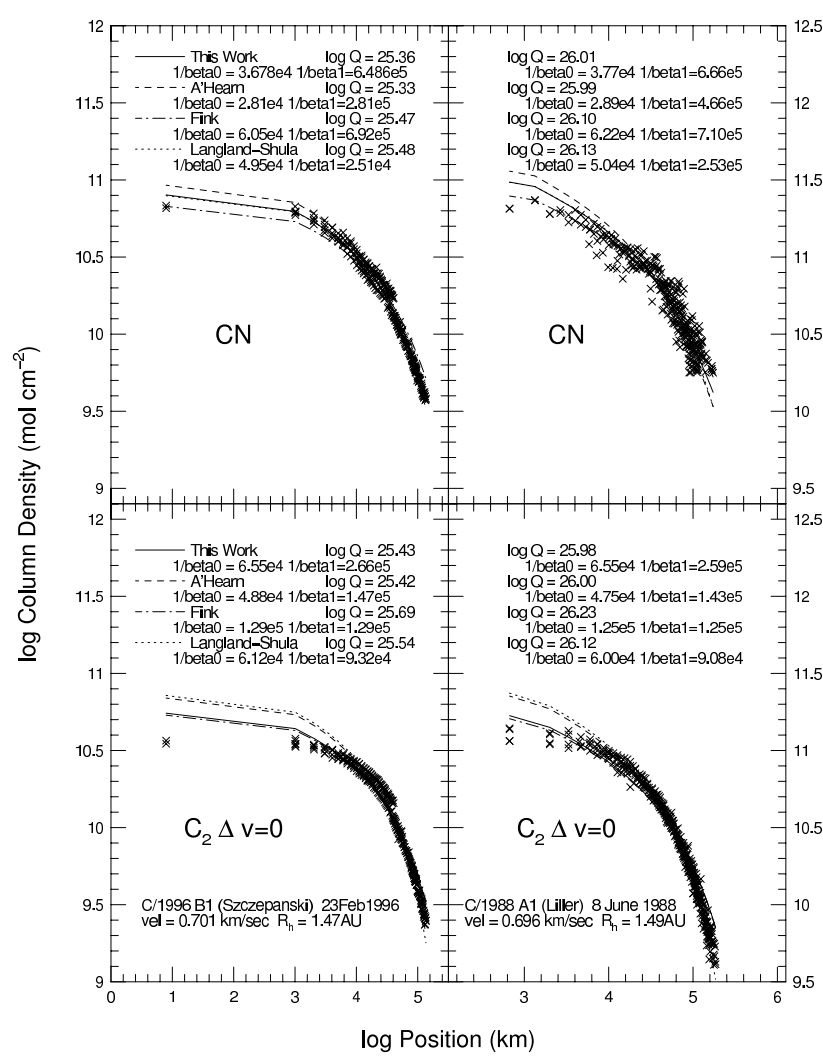

Figure 14: The $\mathrm{CN}$ and $\mathrm{C}_{2}$ data for comets $\mathrm{C} / 1988 \mathrm{~A} 1$ (Liller) and C/1996 B1 (Szczepanski) are shown along with Haser model fits using the scale lengths of this paper, AH95, F09 and LS11. The two comets were observed at comparable heliocentric distances. For $\mathrm{C}_{2}$, the fits using our scale lengths are comparable to those using the scale lengths of F09. The AH95 and LS11 fits are similar. For CN, our fits are indistinguishable from ones using the scale lengths of LS11. The AH95 and F09 models are higher and lower, respectively. However, note the different production rates that are computed with these fits. See text for discussion.

lar cometary data sets were chosen because they are LCS data with lots of points, they are high quality so that the trends are well defined, there is very little asymmetry seen and they are both at approximately the same heliocentric distance. This distance is sufficiently far from $1 \mathrm{AU}$ that the various heliocentric scaling laws play a significant role in the scale lengths. $\mathrm{C}_{2}$ was chosen because it is in the bandpass of all of the surveys. $\mathrm{CN}$ is the molecule used in the denominator of the ratios. Inspection of the two $\mathrm{C}_{2}$ panels shows that our scale lengths do a reasonably good job of fitting the comet Liller data, though they may underestimate the column densities around $10^{4}$ $\mathrm{km}$ from the optocenter. For comet Szczepanski, the fit is good at cometocentric distances greater than $1000 \mathrm{~km}$ but the inner part of the coma is fit poorly. This illustrates that the Haser model does not handle the two-step (grandparent to parent to daughter) dissociation well. The models using the F09 scale lengths are almost indistinguishable in shape to those with our scale lengths, but the derived log production rates differ from ours by $\sim 0.25$. The model fits using the AH95 and LS11 scale lengths are very similar. However, the production rates for the AH95 scale lengths are essentially the same as ours while the LS11 production rates are intermediate to ours and F09. For CN, our model fits and those of LS11 are indistinguishable but yield production rates that differ by 0.12 . Models using F09 and AH95 scale lengths are below and above our model fits, respectively. Again, our production rates match those derived from AH95 scale lengths, while LS11 and F09 scale lengths yield the same, higher, production rates. For Szczepanski, our CN scale lengths fit extremely well, while we overestimate the column densities in the inner coma of Liller.

The scale lengths used for each model are on each panel and are considerably different for the different groups. It is a well known effect that there is a family of Haser model scale 
lengths allowed for each molecule since the parent and daughter scale lengths are degenerate in the model and can compensate for one another. There is nothing in Figure 14 to warrant picking another set of scale lengths over the ones we have used in this paper (Table 2). What is a bit more critical is the boundary for declaring a comet to be typical or depleted. We would say that Liller was slightly depleted. AH95 listed Liller, though not in their restricted set, as typical. Using their scale lengths and our cutoff, we would still find Liller to be depleted. With the AH95 definition and either our or their scale lengths Liller would be typical. We found Szczepanski to be typical, as did F09.

The recent study of LS11 has far fewer comets than our study or the others we compare to. However, the data should be comparable to ours as they used a long slit CCD instrument with a bandpass similar to the LCS. They suffered from difficult sky subtraction because of street lamps near Lick Observatory, something they took great pains to deal with. McDonald does not have this problem. Also, some of their observations were at twilight when the sky contribution was more severe. We almost never had to observe under such conditions so did not encounter these problems.

F09 used a long slit CCD instrument but concentrated on the red region of the spectrum. Thus, he was unable to detect $\mathrm{OH}, \mathrm{NH}$ or $\mathrm{C}_{3}$. For CN, he observed the $\mathrm{CN}$ red system instead of the violet system we observed. F09 observed $\mathrm{NH}_{2}$ and $\mathrm{O}\left({ }^{1} \mathrm{D}\right)$. The latter could be used to derive $\mathrm{Q}\left(\mathrm{H}_{2} \mathrm{O}\right)$.

AH95 used a fundamentally different data type. Their survey used photometry and narrow band filters optimized for comets. With photometry, the apertures are considerably larger than with our spectrograph and so AH95 are much more sensitive at low signal levels. They cannot, however, detect the very weak bands, such as CH. In addition, since the different species are observed sequentially and not simultaneously, they require photometric conditions. Analyses of photometric observations must assume spherical symmetry of the coma since they get little spatial information.

AH95 looked at 85 comets to determine abundance patterns; 41 of those comets formed their restricted data set over which they drew conclusions. They found that 12/41 (29\%) of the comets were in the depleted group. This is significantly more than we found. However, AH96 used a definition based only on a low value for $\mathrm{C}_{2}$. Some of their depleted comets have low $\mathrm{C}_{2}$ but show $\mathrm{C}_{3}$ within normal bounds. This can be seen clearly in their Figure 10, comparing panels $a$ and $b$.

Using only a criterion for depleted comets that $\log \left[Q\left(C_{2}\right) / Q(C N)\right] \leq 0.02$, with no limit on the $\mathrm{C}_{3}$, we add ten comets to our list of depleted comets: 71P/Clark, 26P/GriggSkjellerup, 75P/Kohoutek, C/1988 A1 (Liller), 31P/Schwassmann-Wachmann 2, C/1988 J1 (Shoemaker-Holt), C/1986 V1 (Sorrells), 62P/Tsuchinshan 1, 76P/West-KohoutekIkemura, and C/1989 A1 (Yanaka). With this definition, we find 15/59 depleted comets, or $25 \%$. This is consistent with the quantity found by AH95. These 10 comets all show ratios for $\mathrm{C}_{3}$ production at an average value or below. However, none are close to our defined value for $\mathrm{C}_{3}$ depletion.

AH95 also noted that some comets were enhanced in $\mathrm{C}_{2} / \mathrm{CN}$. We too see such a trend, although not necessarily the same comets. We both found C/1980 Y1 (Bradfield) to be enhanced in $\mathrm{C}_{2}$. In addition, $68 \mathrm{P} /$ Klemola has a strong $\mathrm{C}_{2}$ enhancement. 
F09 did not observe $\mathrm{C}_{3}$ in their bandpass and so their definition of depleted comets was based entirely on the $\mathrm{C}_{2}$ abundance. F09 observed a total of 92 comets, with 50 in their restricted group. F09 broke their comets into more groups than just typical and depleted. However, all comets not in their typical group had $\mathrm{C}_{2}$ depleted relative to $\mathrm{CN}$ (except possibly C/1988 Y1 (Yanaka)). They found 30\% depleted comets.

LS11 observed 26 comets. They did not define a subset of comets as part of a restricted group. Many of their comets were observed on only 1 night. LS11 chose to derive new Haser model scale lengths for their data set because they did not feel that any of the published scale lengths fit their data well. In addition, they derived a heliocentric distance scaling for those scale lengths that was different (not $R_{h}^{2}$ ) than most researchers use. It was not clear if they used mean scale lengths or used different scale lengths for each comet. In addition, it appears from their figures that they did not remove the underlying solar continuum before computing the column densities, though they did fit a continuum before integration. For dusty comets, this will increase the difficulty of detecting the gas. Once they had computed their production rates in this manner, LS11 used the AH95 definition of depleted comets, finding all their Jupiter family comets depleted (including 4P/Faye where they did not detect any $\mathrm{C}_{2}$ and $46 \mathrm{P} /$ Wirtanen that has a much higher $\mathrm{C}_{2} / \mathrm{CN}$ ratio in their data than this cutoff). They also indicated that as a class the long period comets were borderline depleted. LS11 did not attempt to convert the AH95 limits to their scale lengths so it is not obvious that the comets they claim to be depleted would be depleted if they derived a cutoff from their data using their scale lengths. Us- ing the AH95 definition and the data from their Table 11, there are 13/26 comets that are depleted, or $50 \%$ (this includes several with no $\mathrm{C}_{2}$ detection that they take to indicate a comet is depleted).

Table 8 lists the 110 comets we observed that had at least CN detected, along with a summary of our findings. As before, the restricted comets are listed in bold and the remainder are indented. The group of comets that are depleted under our most restrictive definition of low $\mathrm{C}_{2}$ and $\mathrm{C}_{3}$ are noted as "Depleted" (with the word depleted underlined). The additional 10 comets with low $\mathrm{C}_{2}$ but not low $\mathrm{C}_{3}$ are listed as "(depleted)". Comets that have all of their production rate ratios within the normal ranges shown for the restricted set in Table 7 are marked "Typical". There are comets that are not depleted but have at least one production rate ratio outside of the normal ranges. We note the species that are either high or low. Inspection of the table shows that some comets have only one species outside a normal range while others (e.g. C/1990 Y1 (Bradfield)) have many species outside the normal range. Comets with only limits are so noted.

Included in Table 8 are the findings of the other studies discussed above for comets observed in common. When comparing our comets with the findings of others, it is important to remember that only $\mathrm{C}_{2}$ was used in other studies as a criterion for being called depleted, so that any comet where we saw normal $\mathrm{C}_{2}$ but other species out of range would still be typical in other studies.

Inspection of Table 8 shows that we are generally in (remarkably good) agreement on how we classify comets, with some exceptions. As noted above when we examined scale lengths, placement of the cut-off for defining depletion has an important affect on our classifications. 
Table 8: Summary of Abundance Patterns

\begin{tabular}{|c|c|c|c|c|c|}
\hline Comet & $\begin{array}{c}\text { Dynamical } \\
\text { Type }\end{array}$ & $\begin{array}{l}\text { This } \\
\text { Paper }\end{array}$ & $\begin{array}{l}\text { A'Hearn } \\
\text { et al.. } \\
\text { (1995) }\end{array}$ & $\begin{array}{l}\text { Fink } \\
(2009)\end{array}$ & $\begin{array}{c}\text { Langlund-Shula } \\
\text { \& Smith } \\
(2011)\end{array}$ \\
\hline 49P/Arend-Rigaux & JFC & high $\mathrm{NH}, \mathrm{C}_{3}$ & Typical & & \\
\hline C/1982 M1 (Austin) & LPC & Typical & Enhanced & & \\
\hline C/1984 N1 (Austin) & LPC & Typical & Typical & & \\
\hline C/1989 X1 (Austin) & LPC & low NH & Typical & Typical & \\
\hline 19P/Borrelly & JFC & Depleted + low $\mathrm{CH}$ & Depleted & low $\mathrm{C}_{2}$ & \\
\hline 140P/Bowell-Skiff & JFC & low $\mathrm{C}_{3}$, high $\mathrm{CH}$ & & & \\
\hline C/1979 Y1 (Bradfield) & LPC & Depleted & Typical & & \\
\hline C/1980 Y1 (Bradfield) & LPC & high $\mathrm{CH}, \mathrm{C}_{2} ;$ low $\mathrm{NH}_{2}, \mathrm{C}_{3}$ & Enhanced & & \\
\hline C/1987 P1 (Bradfield) & LPC & Typical & Typical & & \\
\hline 16P/Brooks 2 & JFC & Limits & Depleted & & \\
\hline 23P/Brorsen-Metcalf & HTC & high $\mathrm{OH}, \mathrm{CH}$; low $\mathrm{NH}, \mathrm{NH}_{2}$ & Typical & Typical & \\
\hline C/1983 O1 (Cernis) & LPC & high $\mathrm{C}_{3}$ & Depleted & & \\
\hline C/1990 E1 (CKN) & LPC & low $\mathrm{CH}$ & & & \\
\hline C/1980 O1 (CP) & LPC & Limits & Typical & & \\
\hline 67P/CG & $\mathrm{JFC}$ & Typical & Depleted & low $\mathrm{C}_{2}$ & \\
\hline 71P/Clark & $\mathrm{JFC}$ & low $\mathrm{C}_{2}$ (Depleted) & low $\mathrm{C}_{2}$ & & \\
\hline 32P/Comas Sola & JFC & Depleted & & & \\
\hline 27P/Crommelin & HTC & low $\mathrm{NH}_{2}$ & Typical & & \\
\hline 6P/d'Arrest & $\mathrm{JFC}$ & Typical & Typical & Typical & \\
\hline $122 \mathrm{P} / \mathrm{de}$ Vico & HTC & low $\mathrm{C}_{3}$ & Typical & & \\
\hline $79 \mathrm{P} / \mathrm{dTH}$ & $\mathrm{JFC}$ & Limits & & & \\
\hline 2P/Encke & $\mathrm{JFC}$ & low $\mathrm{NH}$, high $\mathrm{CH}$ & Typical & Typical & \\
\hline $4 \mathrm{P} /$ Faye & $\mathrm{JFC}$ & Limits & Depleted & & Depleted \\
\hline $37 \mathrm{P} /$ Forbes & JFC & Limits & & & \\
\hline $78 \mathrm{P} /$ Gehrels 2 & $\mathrm{JFC}$ & Limits & Typical & & \\
\hline $21 P / G Z$ & JFC & $\underline{\text { Depleted }}$ & Depleted & low $\mathrm{C}_{2}, \mathrm{NH}_{2}$ & \\
\hline 84P/Giclas & JFC & Limits & & & \\
\hline $26 P / G S$ & $\mathrm{JFC}$ & low $\mathrm{C}_{2}, \mathrm{NH}_{2}$ (Depleted) & Depleted & & \\
\hline $65 \mathrm{P} /$ Gunn & $\mathrm{JFC}$ & Limits & Depleted & & \\
\hline C/1995 O1 (Hale-Bopp) & LPC & high $\mathrm{C}_{3}, \mathrm{CH}$ & & Typical & Depleted \\
\hline 1P/Halley & HTC & high $\mathrm{CH}, \mathrm{C}_{2}$ & Typical & Typical & \\
\hline 51P/Harrington & JFC & Typical & & & \\
\hline 52P/Harrington-Abell & JFC & Limits & & & \\
\hline C/1995 Q2 (HD) & LPC & Limits & & & \\
\hline 1985 R1 (Hartley-Good) & LPC & high $\mathrm{NH}_{2}$ & Typical & Typical & \\
\hline 161P/Hartley-IRAS & $\mathrm{JFC}$ & high $\mathrm{NH}_{2}$ & Typical & & \\
\hline 111P/HRC & JFC & Limits & & & \\
\hline 45P/HMP & JFC & low $\mathrm{C}_{3}$ & Typical & low $\mathrm{C}_{2}$ & \\
\hline 88P/Howell & JFC & high $\mathrm{C}_{3}$ & Typical & & \\
\hline C/1995 Y1 (Hyakutake) & LPC & low $\mathrm{OH}, \mathrm{NH}$ & & Typical & \\
\hline C/1996 B2 (Hyakutake) & LPC & Typical & & Typical & \\
\hline 153P/Ikeya-Zhang & LPC & low $\mathrm{OH}, \mathrm{C}_{3} ;$ high $\mathrm{CH}$ & & Typical & \\
\hline C/1983 H1 (IAA) & HTC & Typical & Typical & & \\
\hline 126P/IRAS & $\mathrm{JFC}$ & Limits & Depleted & & \\
\hline C/1983 O2 (IRAS) & LPC & Limits & Typical & & \\
\hline 58P/Jackson-Neujmin & JFC & high $\mathrm{NH}, \mathrm{C}_{3}$ & & Typical & \\
\hline 59P/Kearns-Kwee & $\mathrm{JFC}$ & Typical & Typical & & \\
\hline 68P/Klemola & JFC & high $\mathrm{C}_{2}$ & Depleted & & \\
\hline
\end{tabular}


Table 8 continued

\begin{tabular}{|c|c|c|c|c|c|}
\hline Comet & $\begin{array}{c}\text { Dynamical } \\
\text { Type }\end{array}$ & $\begin{array}{c}\text { This } \\
\text { Paper }\end{array}$ & $\begin{array}{l}\text { A'Hearn } \\
\text { et al.. } \\
(1995)\end{array}$ & $\begin{array}{l}\text { Fink } \\
(2009)\end{array}$ & $\begin{array}{l}\text { Langlund-Shula } \\
\text { \& Smith } \\
\text { (2011) }\end{array}$ \\
\hline 75P/Kohoutek & $\mathrm{JFC}$ & low $\mathrm{C}_{2}, \mathrm{NH}_{2}$ (Depleted) & & & \\
\hline 22P/Kopff & JFC & low $\mathrm{OH}$ & Typical & Typical & Depleted \\
\hline 144P/Kushida & JFC & Typical & & & \\
\hline C/1987 A1 (Levy) & LPC & low $\mathrm{CH}$ & & & \\
\hline C/1990 K1 (Levy) & LPC & high $\mathrm{OH}, \mathrm{C}_{3}, \mathrm{C}_{2}$ & Typical & Typical & \\
\hline C/1984 V1 (LR) & LPC & Typical & Typical & & \\
\hline C/1988 A1 (Liller) & LPC & low $\mathrm{C}_{2}$ (Depleted) & Typical & & \\
\hline C/2001 A2 (LINEAR) & LPC & Typical & & & \\
\hline C/2000 WM1 (LINEAR) & LPC & Depleted + low CH & & & \\
\hline C/2006 VZ13 (LINEAR) & LPC & $\overline{\text { low }} \mathrm{OH}$ & & & \\
\hline 93P/Lovas 1 & JFC & high $\mathrm{C}_{3}$ & & Typical & Depleted \\
\hline C/2007 E2 (Lovejoy) & LPC & low $\mathrm{OH}$ & & & Typical \\
\hline C/1994 T1 (Machholz) & LPC & low $\mathrm{NH}$ & & low $\mathrm{C}_{2}$ & \\
\hline C/2004 Q2 (Machholz) & LPC & Typical & & & \\
\hline 141P/Machholz 2-A & JFC & high $\mathrm{CH}$ & & Typical & \\
\hline 115P/Maury & JFC & Limits & & & \\
\hline C/1993 Y1 (MR) & LPC & low $\mathrm{CH}$ & & Typical & \\
\hline C/1980 V1 (Meier) & LPC & low $\mathrm{NH}$ & Typical & & \\
\hline C/1993 A1 (Mueller) & LPC & low $\mathrm{OH}$, high $\mathrm{C}_{3}$ & & & \\
\hline 28P/Neujmin 1 & JFC & high $\mathrm{C}_{3}$ & Typical & & \\
\hline C/1987 B1 (NTT) & LPC & Typical & Typical & & \\
\hline C/1992 W1 (Oshita) & LPC & limits & & & \\
\hline C/1980 Y2 (Panther) & LPC & low $\mathrm{C}_{3}$ & Typical & & \\
\hline 80P/Peters-Hartley & JFC & low $\mathrm{CH}$ & & Typical & \\
\hline 83P/Russell 1 & JFC & limits & & & \\
\hline 91P/Russell 3 & JFC & limits & & & \\
\hline 24P/Schaumasse & JFC & Typical & & Typical & \\
\hline 106P/Schuster & JFC & limits & & & \\
\hline $31 \mathrm{P} / \mathrm{SW} 2$ & JFC & low $\mathrm{C}_{2}$ (Depleted) & Depleted & & \\
\hline 102P/Shoemaker 1 & JFC & high $\mathrm{CH}, \mathrm{NH}_{2}$ & & & \\
\hline C/1984 K1 (Shoemaker) & LPC & high $\mathrm{C}_{3}$ & Enhanced & & \\
\hline C/1984 U2 (Shoemaker) & LPC & limits/Depleted & Depleted & & \\
\hline C/1987 H1 (Shoemaker) & LPC & limits & & & \\
\hline C/1989 A6 (Shoemaker) & LPC & high $\mathrm{C}_{3}$, low $\mathrm{C}_{2}$ (Depleted) & & & \\
\hline C/1988 J1 (SH) & LPC & low $\mathrm{C}_{2}$ (Depleted) & & & \\
\hline 192P/Shoemaker-Levy 1 & JFC & limits & & & \\
\hline C/2002 E2 (SM) & LPC & low $\mathrm{NH}$ & & & \\
\hline C/1986 V1 (Sorrells) & LPC & low $\mathrm{C}_{2}, \mathrm{NH}_{2}$ (Depleted) & Depleted & & \\
\hline 38P/Stephan-Oterma & HTC & high $\mathrm{CH}$ & Typical & & \\
\hline C/1983 J1 (SSF) & LPC & low $\mathrm{C}_{3}$ & Typical & & \\
\hline 64P/Swift-Gehrels & JFC & Typical & & & \\
\hline 109P/Swift-Tuttle & HTC & low $\mathrm{NH}, \mathrm{CH}$ & Typical & Typical & \\
\hline C/1996 B1 (Szczepanski) & LPC & Typical & & Typical & \\
\hline 98P/Takamizawa & JFC & Limits & Depleted & & \\
\hline C/1994 J2 (Takamizawa) & LPC & Typical & & low $\mathrm{C}_{2}$ & \\
\hline C/1994 G1-A (TL) & LPC & Typical & & & \\
\hline 69P/Taylor & JFC & limits & & & \\
\hline 9P/Tempel 1 & JFC & Typical & Typical & low $\mathrm{C}_{2}$ & Depleted \\
\hline
\end{tabular}




\begin{tabular}{|c|c|c|c|c|c|}
\hline Comet & $\begin{array}{c}\text { Dynamical } \\
\text { Type }\end{array}$ & $\begin{array}{l}\text { This } \\
\text { Paper }\end{array}$ & $\begin{array}{l}\text { A'Hearn } \\
\text { et al.. } \\
(1995)\end{array}$ & $\begin{array}{l}\text { Fink } \\
(2009)\end{array}$ & $\begin{array}{c}\text { Langlund-Shula } \\
\text { \& Smith } \\
(2011)\end{array}$ \\
\hline 10P/Tempel 2 & JFC & Typical & Typical & Typical & Depleted \\
\hline 55P/Tempel-Tuttle & HTC & high $\mathrm{CH}$ & & Typical & \\
\hline C/1987 B2 (Terasako) & LPC & low $\mathrm{CH}, \mathrm{C}_{2}$ (Depleted) & & & \\
\hline C1985 T1 (Thiele) & LPC & high $\mathrm{NH}_{2}$ & Typical & & \\
\hline 62P/Tsuchinshan 1 & JFC & low $\mathrm{C}_{2}$ (Depleted) & Typical & Typical & \\
\hline 8P/Tuttle & HTC & low $\mathrm{NH}$ & Typical & & Typical \\
\hline $41 \mathrm{P} / \mathrm{TGK}$ & JFC & high $\mathrm{C}_{3}$ & & & \\
\hline 40P/Vaisala & $\mathrm{JFC}$ & limits & & & \\
\hline 76P/WKI & JFC & high $\mathrm{NH}$, low $\mathrm{C}_{2}$ (Depleted) & & & \\
\hline $81 \mathrm{P} / \mathrm{Wild} 2$ & JFC & Depleted & Depleted & low $\mathrm{C}_{2}$ & Depleted \\
\hline C/1986 P1 (Wilson) & LPC & $\overline{\text { low } \mathrm{NH}_{2}}$ & & & \\
\hline 114P/Wiseman-Skiff & JFC & limits & & & \\
\hline 43P/Wolf-Harrington & JFC & Limits/Depleted & Depleted & low $\mathrm{C}_{2}, \mathrm{NH}_{2}$ & \\
\hline C/1989 A1 (Yanaka) & LPC & low $\mathrm{C}_{2}(\overline{\text { Depleted }})$ & & Typical & \\
\hline
\end{tabular}

In the discussion here, keep in mind our reservations about the definition of depletion used by LS11. In our restricted data set, we find C/1982 M1 (Austin) typical while AH95 find it enhanced. F09 and AH95 find 67P/ChuryumovGerasimenko depleted but we find it typical. C/1995 O1 (Hale-Bopp) is typical in our data and that of F09 but found to be depleted in LS11. AH95 find $68 \mathrm{P} /$ Klemola to be depleted but we find it to be enhanced in $\mathrm{C}_{2}$. 22P/Kopff and 10P/Tempel 2 are typical for us, F09 and AH95, but LS11 find them depleted. 9P/Tempel 1 appears normal to us and AH95, but depleted to F09 and LS11. C/1988 A1 (Liller), 62P/Tsuchinshan 1, and C/1989 A1 (Yanaka) are depleted in our extended definition (Liller and Tsuchinshan 1 barely so) but typical according to AH95 and/or F09. C/1994 T1 (Machholz) is depleted for F09 but typical for us. In addition, there are five comets not in our restricted set where we see some differences with others.

If all of the depleted comets started in the same formation reservoir, we would expect to see all of the depleted comets having the same dynamical type. Of course, the dynamical type does not necessarily correspond to a single reservoir since there have been exchanges of objects from one reservoir to another via gravitational perturbations. For our 59 comets in the restricted data set, 27 are JFCs, 5 are HTCs and 27 are LPCs. Using our strict definition of carbonchain depleted comets, we find $4 / 5$ of them are JFCs (80\%) and 1 is an LPC (20\%). Using the more relaxed definition requiring only $\mathrm{C}_{2}$ to be depleted, we find 10 of the depleted comets are JFCs $(67 \%)$ and 5 are LPCs $(33 \%)$. For a particular dynamical type and the relaxed definition, $10 / 27$ or $37 \%$ of the JFCs are depleted; $5 / 27$ or $18.5 \%$ of the LPCs are depleted. We see no depleted HTCs, but with only 5 HTCs in our sample this might not be significant.

In Table 9, we show the average production rate ratios as a function of dynamical type, combining depleted and typcial comets in each dynamical type. Inspection of Table 9 shows that the averages are indistinguishable for $\mathrm{OH}$ (with only 1 Halley Type comet with $\mathrm{OH}$ the deviation of this group is not meaningful), or $\mathrm{C}_{3}$. $\mathrm{CH}$ and $\mathrm{NH}_{2}$ might show differences with dynami- 
Table 9: Production Rate Ratios as a function of Dynamical Type

\begin{tabular}{ccccccc}
\hline & \multicolumn{5}{c}{ Log Production Rate Ratio with respect to CN } \\
\cline { 2 - 7 } & $\mathrm{OH}$ & $\mathrm{NH}$ & $\mathrm{C}_{3}$ & $\mathrm{CH}$ & $\begin{array}{c}\mathrm{C}_{2} \\
\text { (average) }\end{array}$ & $\mathrm{NH}_{2}$ \\
\hline Jupiter Family & $2.15 \pm 0.32$ & $0.60 \pm 0.24$ & $-0.69 \pm 0.25$ & $0.11 \pm 0.19$ & $0.04 \pm 0.31$ & $-0.03 \pm 0.28$ \\
$(27$ comets $)$ & $(11$ comets $)$ & $(12$ comets $)$ & $(27$ comets $)$ & $(14$ comets $)$ & $(27$ comets $)$ & $(11$ comets $)$ \\
Halley Type & 2.76 & 0.25 & $-0.67 \pm 0.08$ & $0.46 \pm 0.28$ & $0.16 \pm 0.13$ & $0.10 \pm 0.34$ \\
$\quad(5$ comets $)$ & $(1$ comet $)$ & $(1$ comet $)$ & $(5$ comets $)$ & $(4$ comets $)$ & $(5$ comets $)$ & $(4$ comets $)$ \\
Long Period & $2.17 \pm 0.38$ & $0.36 \pm 0.18$ & $-0.68 \pm 0.12$ & $0.27 \pm 0.17$ & $0.18 \pm 0.11$ & $-0.17 \pm 0.22$ \\
$(27$ comets $)$ & $(11$ comets $)$ & $(14$ comets $)$ & $(27$ comets $)$ & $(23$ comets $)$ & $(27$ comets $)$ & $(11$ comets $)$ \\
\hline
\end{tabular}

cal type but the error bars are large (and overlapping) and there is no obvious sequence with current orbit size. For NH, there appears to be a significant difference between JFCs and LPCs (again HTCs do not have enough data to be meaningful) but the error bars are overlapping. For $\mathrm{C}_{2}$, there is a significant difference between JFCs and other dynamical types, though LPCs and HTCs are identical. This trend for $\mathrm{C}_{2}$ is consistent with us finding many more JFCs that are depleted and is in agreement with the trend found by AH95. We concur with their conclusion that the compositional similarities between dynamical types are suggestive that the interiors of comets are similar to their exteriors. In contrast, LS11 reported a trend that the $\mathrm{C}_{2}, \mathrm{NH}$ and $\mathrm{NH}_{2}$ increased in a regular fashion as the comet orbits became larger (though their Table 12 shows the $\mathrm{C}_{2} / \mathrm{CN}$ decreasing from HTCs to LPCs and being the same for dynamically new and HTCs).

Perhaps the differences we see for a large number of JFCs is the result of evolutionary differences instead of formational differences. While we cannot categorically refute this concept, we point to observations of comet 73P/Schwassmann-Wachmann 3 (SW3) as evidence to the contrary. Comet SW3 split into several pieces in 1995. It made a close ap- proach to the Earth in 2003 and was extensively studied. 73P/Schwassmann-Wachmann 3 showed no compositional differences between different pieces (Kobayashi et al. 2007, Jehin et al. 2008, Weaver et al. 2008, Schleicher and Bair 2011), suggesting that the comet was the same composition throughout. SW3 is a JFC that is depleted. Therefore, its depletion is not just a surface effect.

AH95 found a trend of dust-to-gas ratio as a function of cometary perihelion distance (their Figure 4). With our small apertures, we do not feel comfortable computing A $f \rho$ so cannot comment on whether our data show such a trend. We did, however, examine the production rate ratios as a function of cometary perihelion distance. These are shown in Figure 15. We find no believable trend of the production rate ratios with perihelion distance.

\section{Summary}

When we began this survey in 1980, we fully expected that we would find some comets with very different spectra than the vast majority of comets. Instead, we found a mostly homogeneous group of objects with subtle compositional differences. In particular, we found: 


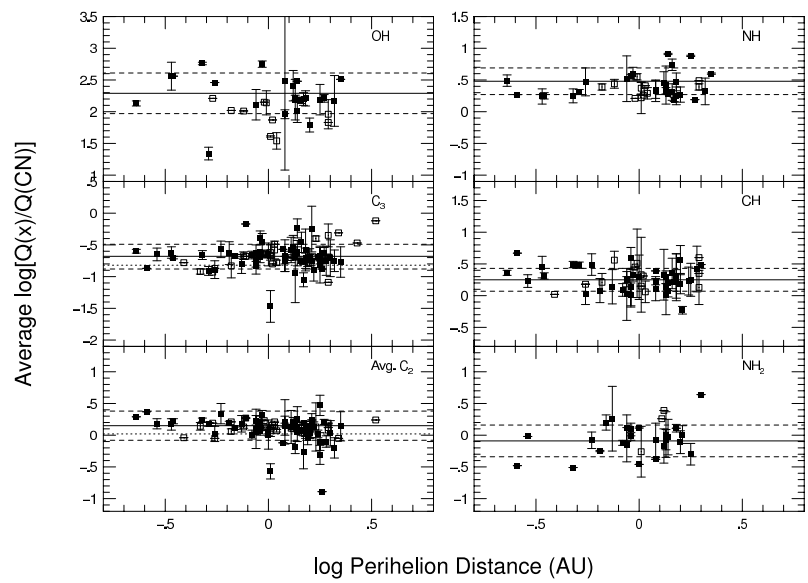

Figure 15: The production rate ratios are plotted as a function of the perihelion distances of the current cometary orbits. The symbols used are the same as in Figure 12 as are the definitions of the horizontal lines. There are no believable trends of the ratios as a function of perihelion distance.

- The vast majority of comets have remarkably similar compositions.

- There exists a group of comets which are depleted in the carbon-chain molecules. Using a very strict definition of carbon-chain depletion requiring depletion in both $\mathrm{C}_{2}$ and $\mathrm{C}_{3}$, we find that $9 \%$ of the comets are depleted. Using a more relaxed definition that requires only the $\mathrm{C}_{2}$ be depleted (similar to other authors), we find $25 \%$ of the comets are depleted. This is consistent with other authors.

- For the most part, we agree with other studies on which individual comets show depletions.

- Carbon-chain depleted comets can be of any dynamical type (though we did not observe any HTCs with depletion our HTC sample is very small).
- Using the relaxed definition, two thirds of the depleted comets are JFCs and one third are LPCs (with the stricter definition it is $80 \%$ and $20 \%$ respectively).

- Depleted comets make up $37 \%$ of the JFCs and $18.5 \%$ of the LPCs.

- On average, there are few differences in composition with increasing current orbit semimajor axis. However, $\mathrm{C}_{2}$ and $\mathrm{NH}$ may show significant trends of lower $\mathrm{C}_{2}$ and higher $\mathrm{NH}$ for JFCs than for LPCs.

\section{Acknowledgments}

This research was supported by NASA Grants NNG04G162G, NNX08A052G and predecessor grants. McDonald Observatory is operated by The University of Texas at Austin. We thank David Schleicher for performing a cluster analysis for us that, unfortunately, did not clarify the groupings. We thank Dr. Michael A'Hearn and Dr. Uwe Fink for very helpful suggestions to clarify the paper and for identifying some errors.

\section{References}

[1] Ádámkovics, M., G. A. Blake, and B. J. MCCALL 2003. Observations of rotationally resolve $\mathrm{C}_{3}$ in translucent sight lines. Ap. J. 595, 235-246.

[2] A'Hearn, M. F., P. V. Birch, P. FeldMAN, AND R. L. MiLlis 1985. Comet Encke: Gas production and lightcurve. Icarus $\mathbf{6 4}$, $1-10$. 
[3] A'Hearn, M. F. And R. L. Millis 1980. Abundance correlations among comets. A. J. 85, 1528-1537.

[4] A'Hearn, M. F., R. L. Millis, D. G. Schleicher, D. J. Osip, AND P. V. Birch 1995. The ensemble properties of comets: Results from narrowband photometry of 85 comets, 1976-1992. Icarus 118, 223-270.

[5] Arvesen, J. C., R. J. Griffin, JR., And B. D. Pearson, JR. 1969. Determination of extraterrestrial Solar spectral irradiance from a research aircraft. Appl. Opt. 8, 2215-2232.

[6] Clegga, R. E. S. and D. L. Lambert 1982. On $\mathrm{c}_{3}$ molecules in diffuse interstellar clouds. M.N.R.A.S. 201, 723-733.

[7] Cochran, A. L. 1986. A reevaluation of the Haser model scale lengths in comets. A. J. 90, 2609-2614.

[8] Cochran, A. L. 1987. Another look at abundance correlations among comets. A. J. 93, 231-238.

[9] Cochran, A. L. And E. S. Barker 1985. Spatially resolved spectrophotometry of comet P/Stephan-Oterma. Icarus 62, 72-81.

[10] Cochran, A. L. And E. S. Barker 1987. Comet Giacobini-Zinner: A normal comet? A. J. 93, 239-243.

[11] Cochran, A. L., E. S. Barker, M. D. Caballero, and J. Györgey-Ries 2009. Placing the Deep Impact mission into context: Two decades of observations of $9 \mathrm{P} /$ Tempel 1 from McDonald Observatory. Icarus 199, 119-128.
[12] Cochran, A. L., E. S. Barker, And W. D. Cochran 1980. Spectrophotometric observations of $\mathrm{P} /$ Schwassmann-Wachmann 1 during outburst. A. J. 85, 474-477.

[13] Cochran, A. L., E. S. Barker, T. F. RAmseyer, AND A. D. Storrs 1992. The McDonald Observatory Faint Comet Survey: Gas production in 17 comets. Icarus 98, 151162.

[14] Cochran, A. L. And W. D. Cochran 1990. Observations of $\mathrm{CH}$ in comets $\mathrm{P} /$ Brorsen-Metcalf and $\mathrm{P} /$ Halley. In Workshop on Observations of Recent Comets (1990) (W. F. Huebner, P. A. Wehinger, J. Rahe, and I. Konno, Eds.) pp. 22-27 Southwest Research Institute San Antonio, TX.

[15] Cochran, A. L. And W. D. Cochran 1991. The first detection of $\mathrm{CN}$ and the distribution of $\mathrm{CO}^{+}$gas in the coma of comet $\mathrm{P} /$ Schwassmann-Wachmann 1. Icarus 90, 172-175.

[16] Cochran, A. L., W. D. Cochran, AND E. S. BARKer 1982. Spectrophotometry of comet Schwassmann-Wachmann 1. II. Its color and $\mathrm{CO}^{+}$emission. Ap. J. 254, 816-822.

[17] Cochran, A. L., W. D. Cochran, E. S. Barker, And A. D. Storrs 1991. The development of the $\mathrm{CO}^{+}$coma of comet $\mathrm{P} /$ Schwassmann-Wachmann 1. Icarus 92, 178-183.

[18] Cochran, A. L., J. R. Green, and E. S. BARKER 1989. Are low activity comets different from more active comets? Icarus $\mathbf{7 9}$, 125-144 plus erratum Icarus 80, 446, 1989. 
[19] Cochran, A. L. And D. G. Schleicher 1993. Observational constraints on the lifetime of cometary $\mathrm{H}_{2} \mathrm{O}$. Icarus 105, 235-253.

[20] Cochran, A. L., A. L. Whipple, P. J. MacQueen, P. J. Shelus, R. W. Whited, AND C. F. Claver 1994. Pre-impact characterization of comet Shoemaker-Levy 9. Icarus 112, 528-532.

[21] Cochran, A. L. And B. D. White 1993. Spectroscopic observations of $\mathrm{CO}^{+}$in P/Schwassmann-Wachmann 1: 1978-1991. In Proceedings of the Lenggries Workshop on the Activity of Distant Comets (W. F. Huebner, H. U. Keller, D. Jewitt, J. Klinger, and R. West, Eds.) pp. 29-38 Southwest Research Institute, San Antonio, TX.

[22] Combi, M. R. AND U. Fink 1993. P/Halley: Effects of time-dependent production rates on spatial emission profiles. Ap. J. 409, 790-797.

[23] Fink, U. 1994. The trend of production rates with heliocentric distance for comet P/Halley. Ap. J. 423, 461-473.

[24] Fink, U. 2009. A taxonomic survey of comet composition 1985 - 2004 using CCD spectroscopy. Icarus 201, 311-334.

[25] Fink, U. And M. D. Hicks 1996. A survey of 39 comets using CCD spectroscopy. Ap. J. 459, 720-743.

[26] Haser, L. 1957. Distribution d'intensité dans la têtê d'une comète. Liege Inst. Astrophysics Reprint No. 394.

[27] Ihalawela, C. A., D. M. Pierce, G. R. Dorman, And A. L. Cochran 2011. The spatial distribution of $\mathrm{OH}$ and $\mathrm{CN}$ radicals in the coma of comet Encke. Ap. J. 741, 89.

[28] Jehin, E., J. Manfroid, H. Kawakita, D. Hutsemékers, M. Weiler, C. Arpigny, A. Cochran, O. HainAut, H. Rauer, R. Schulz, And J.-M. ZuCCONI 2008. Optical spectroscopy of the B and $\mathrm{C}$ fragments of comet $73 \mathrm{P} /$ SchwassmannWachmann 3 at the ESO VLT. LPI Contributions 1405, p.8319.

[29] Jørgensen, U. G., J. AlmlöF, And P. E. M. Siegbahn 1989. Complete active space self-consistent field calculations of the vibrational band strengths for $\mathrm{C}_{3} . A p . J$. 343, 554-561.

[30] Kim, S. J., M. F. A'Hearn, and W. D. CoCHRAN 1989. NH emissions in comets: Fluorescence vs collisions. Icarus $\mathbf{7 7}, 98-108$.

[31] Kobayashi, H., H. Kawakita, M. J. Mumma, B. P. Bonev, J.-I. Watanabe, AND T. Fuse 2007. Organic volatiles in comet 73P-B/Schwassmann-Wachmann 3 observed during its outburst: A clue to the formation region of the Jupiter-Family comets. Ap. J.(Letters) 668, L75-L78.

[32] Langland-Shula, L. E. And G. H. SMITH 2011. Comet classification with new methods for gas and dust spectroscopy. Icarus 213, 280-322.

[33] Newburn, R. L. And H. Spinrad 1984. Spectrophotometry of 17 comets. I. The emission features. A. J. 89, 289-309.

[34] Oliversen, R. J., J. M. Hollis, And L. W. Brown 1985. C $\mathrm{C}_{2}$ imagery of the inner coma of comet Iras-Araki-Alcock. Icarus 63, 339-346. 
[35] Randall, C. E., D. G. Schleicher, R. G. Ballou, and D. J. Osip 1992. Observational constraints on molecular scalelengths and lifetimes in comets. Bull. AAS 24, 1002.

[36] Roueff, E., P. Felenbok, J. H. Black, AND C. GRY 2002. Interstellar $\mathrm{C}_{3}$ toward HD 210121. Astr. and Ap. 384, 629-637.

[37] Schleicher, D. G. 2010. The fluorescence efficiencies of the $\mathrm{CN}$ violet bands in comets. A. J. 140, 973-984.

[38] Schleicher, D. G. And M. F. A'Hearn 1982. OH fluorescence in comets: Fluorescence efficiency of the ultraviolet bands. Ap. J. 258, 864-877.

[39] Schleicher, D. G. AND A. N. Bair 2011. The composition of the interior of comet 73P/Schwassmann-Wachmann 3: Results from narrowband photometry of multiple components. A. J. 141, 177-.

[40] Stone, R. P. S. 1977. Spectral energy distributions of standard stars of intermediate brightness. II. Ap. J. 218, 767-769.

[41] Tatum, J. B. and M. J. Gillespie 1977. The cyanogen abundance of comets. Ap. J. 218, 569-572.

[42] Tegler, S. And S. Wyckoff 1989. $\mathrm{NH}_{2}$ fluorescence efficiencies and the $\mathrm{NH}_{3}$ abundance in comet Halley. Ap. J. 343, 445-449.

[43] Weaver, H. A., C. M. Lisse, M. Mutchler, P. L. Lamy, I. Toth, W. T. Reach, and J. VAubaillon 2008. Hubble investigation of the $B$ and $G$ fragments of comet 73P/Schwassmann-Wachmann 3 . LPI Contributions, 1405, p. 8248.
[44] Wing, R. F. 1967. Infrared Spectrophotometry of Red Giant Stars. PhD thesis U. C. Berkeley. 